\title{
Verified Quadratic Virtual Substitution for Real Arithmetic
}

\author{
Matias Scharager Katherine Cordwell Stefan Mitsch André Platzer*
}

\begin{abstract}
This paper presents a formally verified quantifier elimination (QE) algorithm for first-order real arithmetic by linear and quadratic virtual substitution (VS) in Isabelle/HOL. The Tarski-Seidenberg theorem established that the first-order logic of real arithmetic is decidable by QE. However, in practice, QE algorithms are highly complicated and often combine multiple methods for performance. VS is a practically successful method for QE that targets formulas with low-degree polynomials. To our knowledge, this is the first work to formalize VS for quadratic real arithmetic including inequalities. The proofs necessitate various contributions to the existing multivariate polynomial libraries in Isabelle/HOL. Our framework is modularized and easily expandable (to facilitate integrating future optimizations), and could serve as a basis for developing practical general-purpose QE algorithms. Further, as our formalization is designed with practicality in mind, we export our development to SML and test the resulting code on 378 benchmarks from the literature, comparing to Redlog, Z3, Wolfram Engine, and SMT-RAT. This identified inconsistencies in some tools, underscoring the significance of a verified approach for the intricacies of real arithmetic.
\end{abstract}

\section{Introduction}

Quantifier elimination $(Q E)$ is the process of transforming quantified formulas into logically equivalent quantifier-free formulas. In this paper, we consider $\mathrm{QE}$ for the first-order logic of real arithmetic $\left(\mathrm{FOL}_{\mathbb{R}}\right)$, so quantifiers range over the real numbers. The Tarski-Seidenberg theorem proves that $\mathrm{QE}$ is admissible for the theory of real-closed fields [25, 29]. Real quantified statements arise in a number of application domains, including geometry, chemistry, life sciences, and the verification of cyber-physical systems (CPS) [27]. Many of the applications which require QE are safety-critical [19.20]; accordingly, it is crucial to have both efficient and formally verified support for QE to trust the resulting decisions.

Unfortunately, QE algorithms are quite intricate, which makes it difficult to formally verify their correctness. In practice, this necessitates the use of unverified tools. For example, the theorem prover KeYmaera X [10], which is designed to formally verify models of CPS (such as planes and surgical robots) uses Mathematica/Wolfram Engine and/or Z3 as blackbox solvers for QE. While these are admirable tools, they are unverified, and their use introduces a weak link [8] into what would otherwise be a (fully verified [1]) trustworthy proof.

To help fill this gap, we formally verify linear and quadratic virtual substitution (VS) due to Weispfenning [30, 32], which focuses on QE for a quantified variable $x$ occurring in polynomials $f(x)$ of at most degree 2 in $x$, although variations [14,31] handle higher degree polynomials. Linear and quadratic VS are of

\footnotetext{
${ }^{*}$ Computer Science Department, Carnegie Mellon University, Pittsburgh, USA \{mscharag,kcordwel,smitsch,aplatzer\}@cs.cmu.edu
} 
practical significance. They serve to improve QE [18] and SMT tools and are the basis of the experimentally successful [28] Redlog solver [7]. To our knowledge, ours is the first formally verified algorithm for VS with quadratic inequalities.

As we focus on correct and practical VS, we export our verified Isabelle/HOL code to SML for experimentation. We test our exported formalization of the equality VS algorithm (Sect.3.2) and of the general VS algorithm (Sect. 3.3). We compare to four tools that implement real QE: Redlog, SMT-RAT [5], Z3 [6], and Wolfram Engine. With 304 examples, we solve more examples than SMT-RAT in quantifier elimination mode (solves 191) and come close to virtual substitution in Wolfram Engine (solves 322). The remaining tools solve almost all examples; this is to be expected given that those tools have been optimized and fine-tuned (some for decades) and use efficient general-purpose fallback QE algorithms when VS does not succeed. However, as we found 137 inconsistencies in other solvers, it is significant that ours is the only VS implementation with associated correctness proofs (assuming the orthogonal challenge of correct code generation from Isabelle [12]).

Our formalization is approximately 23,000 lines in Isabelle/HOL and is available on the Archive of Formal Proofs (AFP) [23].

\section{Related Work}

The fastest known QE algorithm is Cylindrical Algebraic Decomposition (CAD) [4], which has not yet been fully formally verified. There are few general-purpose formally verified QE algorithms, and there appears to exist a tradeoff between the practicality of an algorithm and the ease of formalization. Mahboubi and Cohen verified Tarski's original QE algorithm [3] and McLaughlin and Harrison have a proof-producing QE procedure based on Cohen-Hörmander [15]; unfortunately, Tarski's algorithm and Cohen-Hörmander both have non-elementary complexity, which limits the computational feasibility of these formalizations.

There has already been some work on formally verified VS: Nipkow [17] formally verified a VS procedure for linear equations and inequalities. The building blocks of $\mathrm{FOL}_{\mathbb{R}}$ formulas, or "atoms," in Nipkow's work only allow for linear polynomials $\sum_{i} a_{i} x_{i} \sim c$, where $\sim \in\{=,<\}$, the $x_{i}$ 's are quantified variables and $c$ and the $a_{i}$ 's are real numbers. These restrictions ensure that linear QE can always be performed, and they also simplify the substitution procedure and associated proofs. Nipkow additionally provides a generic framework that can be applied to several different kinds of atoms (each new atom requires implementing several new code theorems in order to create an exportable algorithm). While this is an excellent theoretical framework-we utilize several similar constructs in our formulation-we create an independent formalization that is specific to general $\mathrm{FOL}_{\mathbb{R}}$ formulas, as our main focus is to provide an efficient algorithm in this domain. Specializing to one type of atom allows us to implement several optimizations, such as our modified DNF algorithm, which would be unwieldy to develop in a generic setting.

Chaieb [2] extends Nipkow's work to quadratic equalities. His formalizations are not publicly available, but he generously provided us with the code. While this was helpful for reference, we chose to build on a newer Isabelle/HOL polynomial library, and we focus on VS as an exportable standalone procedure, whereas Chaieb intrinsically links VS with an auxiliary QE procedure.

Other related work includes some unverified solvers. For example, some work has been done in constraint solving with falsification: RSolver [22] was designed for hybrid systems verification and can find concrete counterexamples for fully quantified existential QE problems on compact domains. dReal [11] is based on similar ideas and slightly relaxes the notion of satisfiability to $\delta$-satisfiability. Constraint solving has also been considered in SMT-solving with Z3's nlsat [13], which uses CDCL to decide systems of nonlinear inequalities and equations. 


\section{The Virtual Substitution Algorithm}

Informally (and broadly) speaking, VS discretizes the QE problem by solving for the roots of one or more low-degree polynomials $f_{1}(x), \ldots, f_{n}(x)$. VS focuses on these roots and the intervals around them to identify and substitute appropriate representative "sample points" for $x$ into the rest of the formula. However, these sample points may contain fractions, square roots, and/or other extensions of the logical language, and so they must be substituted "virtually": That is, VS creates a formula in $F O L_{\mathbb{R}}$ proper that models the behavior of the direct substitution, which would be outside of FOL $\mathrm{R}_{\mathbb{R}}$. VS applies in two cases: an equality case and a general case. We formalize both, and discuss each in turn.

Remark 1 The VS algorithms need to work for multivariate polynomials. But as the VS correctness proofs show the equivalence is true for every real value of the free variables, they often implicitly treat all but one variable as having fixed (but arbitrary) real values. That is why most correctness lemmas (but not the top-level algorithmic constructions) suffice for univariate polynomials with real coefficients. We utilize this trick to simplify difficult proofs for general VS.

\subsection{Example}

Example 1 Say that we want to perform $Q E$ on the formula $\exists x \cdot\left(x^{2}=2 \wedge x y^{2}+2 y+1=0\right)$. One might notice that $x^{2}=2$ forces $x= \pm \sqrt{2}$ and accordingly wish to substitute. Direct substitution yields the following expression: $\left(\sqrt{2} y^{2}+2 y+1=0 \vee-\sqrt{2} y^{2}+2 y+1=0\right)$. However, as its mention of the $\sqrt{ }$. operator makes it an illegal $F O L_{\mathbb{R}}$ formula, we will need some further tricks.

Cleverly, VS finds that $\sqrt{2} y^{2}+2 y+1=0$ is logically equivalent to $y^{2} \cdot(2 y+1) \leq 0 \wedge 2 y^{4}-(2 y+$ $1)^{2}=0$, which is a FOL $L_{\mathbb{R}}$ formuld 1 Similarly, VS identifies a $F O L_{\mathbb{R}}$ formula that is logically equivalent to $-\sqrt{2} y^{2}+2 y+1=0$. Then, VS returns the following quantifier-free $F O L_{\mathbb{R}}$ formula which is logically equivalent to $\exists x .\left(x^{2}=2 \wedge x y^{2}+2 y+1=0\right)$ :

$$
\begin{aligned}
& \left(\left(y^{2} \cdot(2 y+1) \leq 0 \wedge 2 y^{4}-(2 y+1)^{2}=0\right)\right. \\
& \left.\vee\left(-y^{2} \cdot(2 y+1) \leq 0 \wedge 2 y^{4}-(2 y+1)^{2}=0\right)\right)
\end{aligned}
$$

Remark 2 If instead our starting formula were $\exists x \cdot \exists y \cdot\left(x^{2}=2 \wedge x y^{2}+2 y+1=0\right)$, where now $y$ is quantified, then (following the same method as above) VS would identify the following logically equivalent $F O L_{\mathbb{R}}$ formula with fewer variables:

$$
\begin{aligned}
& \exists y \cdot\left(\left(y^{2} \cdot(2 y+1) \leq 0 \wedge 2 y^{4}-(2 y+1)^{2}=0\right)\right. \\
& \left.\quad \vee\left(-y^{2} \cdot(2 y+1) \leq 0 \wedge 2 y^{4}-(2 y+1)^{2}=0\right)\right) .
\end{aligned}
$$

Unfortunately, here we are left with a quantified formula with no linear or quadratic equations or inequalities. As we are thus outside of the fragment of $F O L_{\mathbb{R}}$ that standard VS applies to, at this point we would want to outsource (1) to a general-purpose $Q E$ algorithm (like CAD) to eliminate the quantifier on $y$.

Example 1 was relatively simple, because it involved a quadratic equation with constant coefficients for $x$. However, nothing in our reasoning was limited to constant coefficients: To perform QE on $\exists x .\left(x^{2}=\right.$

\footnotetext{
${ }^{1}$ Notice that if $y=0$, then both $\sqrt{2} y^{2}+2 y+1=0$ and $y^{2} \cdot(2 y+1) \leq 0 \wedge 2 y^{4}-(2 y+1)^{2}=0$ are false. If instead $y \neq 0$, then $\sqrt{2} y^{2}+2 y+1=0$ is true exactly when $\sqrt{2}=-(2 y+1) / y^{2}$, or exactly when $-(2 y+1) / y^{2} \geq 0 \wedge 2 y^{4}-(2 y+1)^{2}=0$, which is logically equivalent to $y^{2} \cdot(2 y+1) \leq 0 \wedge 2 y^{4}-(2 y+1)^{2}=0$, as desired.
} 
$c \wedge x y^{2}+2 y+1=0$ ), where $c$ is a polynomial in the variable $z$, we could handle substituting $x= \pm \sqrt{c}$ in the exact same way as for $x= \pm \sqrt{2}$, but the answer must distinguish the case of $c \geq 0$ symbolically. More difficult is the generalization to inequalities, which seemingly require uncountably infinitely many values to be virtually substituted. We first turn to the general equality case, and then discuss inequalities.

\subsection{Equality Virtual Substitution Algorithm}

Let $a, b$ and $c$ be arbitrary polynomials with real coefficients that do not mention the variable $x$. Consider the formula $\exists x .\left(a x^{2}+b x+c=0 \wedge F\right)$. There are three possible cases: Either $a \neq 0$, or $a=0$ and $b$ is nonzero, or all of $a, b, c$ are zero (so $a x^{2}+b x+c=0$ is uninformative). Letting $F_{x}^{r}$ denote the substitution of $x=r$ for $x$ in $F$, and solving for the roots of $a x^{2}+b x+c$, we have the following:

$$
\begin{aligned}
& \exists x .\left(a x^{2}+b x+c=0 \wedge F\right) \longleftrightarrow \\
& ((a=0 \wedge b=0 \wedge c=0 \wedge \exists x . F) \vee \\
& \left(a=0 \wedge b \neq 0 \wedge F_{x}^{-c / b}\right) \vee \\
& \left.\left(a \neq 0 \wedge b^{2}-4 a c \geq 0 \wedge\left(F_{x}^{\left(-b+\sqrt{b^{2}-4 a c}\right) /(2 a)} \vee F_{x}^{\left(-b-\sqrt{b^{2}-4 a c}\right) /(2 a)}\right)\right)\right) .
\end{aligned}
$$

Conditions such as $b^{2}-4 a c \geq 0$ are needed to ensure $\left(-b \pm \sqrt{b^{2}-4 a c}\right) /(2 a)$ are well-defined; these are symbolic formulas unless $a, b, c$ are concrete numbers.

Similarly as in Example 1 , if we were to substitute $F_{x}^{-c / b}, F_{x}^{\left(-b+\sqrt{b^{2}-4 a c}\right) /(2 a)}$, and $F_{x}^{\left(-b-\sqrt{b^{2}-4 a c}\right) /(2 a)}$ directly (for polynomials $a, b$, and $c$ that do not involve $x$ ), the resulting formula would no longer be in $\mathrm{FOL}_{\mathbb{R}}$. Instead, VS avoids directly dividing polynomials or taking square roots with equivalent rewritings in $\mathrm{FOL}_{\mathbb{R}}$. This involves two procedures: one for fractions, and one for square roots.

To virtually substitute a fraction $p / q$ of polynomials where $q \neq 0$ into the atom $\sum_{i=0}^{n} a_{i} x^{i} \sim 0$, where $\sim \in\{=,<, \leq, \neq\}$ and each $a_{i}$ is an arbitrary polynomial expression not involving $x$, it suffices to normalize the denominator of the LHS, with the caveat that we must not flip the direction of the inequality for $<$ and $\leq$ atoms by normalizing by a value that might be negative. When $n$ is even, $q^{n} \geq 0$ under any possible valuation, so normalizing by $q^{n}$ does not flip the inequality. Alternatively, if $n$ is odd, $q^{n+1} \geq 0$. We formalize this in our linear_substitution function (see Appendix A.1.).

Next, we consider substituting $x=\sqrt{c}$ into an atom $\sum_{i=0}^{n} a_{i} x^{i} \sim 0$, where $c$ is an arbitrary polynomial expression not involving $x$ that satisfies $c \geq 0$, each $a_{i}$ is an arbitrary polynomial expression not involving $x$, and $\sim \in\{=,<, \leq, \neq\}$. Its direct substitution can be separated out into even and odd exponents:

$$
\sum_{i=0}^{n} a_{i} \cdot(\sqrt{c})^{i}=\sum_{i=0}^{n / 2} a_{2 i} c^{i}+\sum_{i=0}^{n / 2} a_{2 i+1} c^{i} \sqrt{c}
$$

Now our polynomial has the form $A+B \sqrt{c}$, where $A$ and $B$ and $c$ are symbolic polynomial expressions not involving $x$. Then, we have the following cases:

$$
\begin{aligned}
& A+B \sqrt{c}=0 \longleftrightarrow A B \leq 0 \wedge A^{2}-B^{2} c=0 \\
& A+B \sqrt{c}<0 \longleftrightarrow\left(A<0 \wedge B^{2} c-A^{2}<0\right) \vee\left(B \leq 0 \wedge\left(A<0 \vee A^{2}-B^{2} c<0\right)\right) \\
& A+B \sqrt{c} \leq 0 \longleftrightarrow\left(A \leq 0 \wedge B^{2} c-A^{2} \leq 0\right) \vee\left(B \leq 0 \wedge A^{2}-B^{2} c \leq 0\right) \\
& A+B \sqrt{c} \neq 0 \longleftrightarrow-A B<0 \vee A^{2}-B^{2} c \neq 0
\end{aligned}
$$


The equivalences for $=$ and $\neq$ atoms are derived from the observation that if $B \neq 0, A+B \sqrt{c}=0$ can be solved to find $\sqrt{c}=-A / B$, which holds iff $A^{2}=B^{2} c$ and $-A / B \geq 0$. The inequality cases involve casework to determine when polynomial $A$ is negative and dominates $B \sqrt{c}$ as $A^{2}>B^{2} c$, and when $B$ is negative and $B \sqrt{c}$ dominates $A$ as $B^{2} c>A$. We formalize the VS procedure for quadratic roots in quadratic_sub (see Appendix A.2.).

\subsection{General Virtual Substitution Algorithm}

As we have seen, QE very naturally leads to finitely many cases (discretizes) for formulas that involve quadratic equality atoms (we call this the equality case). The VS algorithm for the general case, which also handles inequality atoms, is more involved, because, unlike equalities, inequalities may have uncountably many solutions. General VS only directly applies to a very specific fragment of $\mathrm{FOL}_{\mathbb{R}}$ formulas: conjunctions of polynomials that are at most quadratic in the variable of interest. However, we can extend general VS to apply to more formulas with the help of a disjunctive normal form (DNF) transformation.

As a simple example, consider the formula $\exists x .(p<0 \wedge q<0)$, where $p$ and $q$ are the univariate quadratic polynomials (in variable $x$ ) depicted in Fig. 1. Noting that the roots of $p$ and $q$ cannot possibly satisfy the strict inequalities, we partition the number line in

We recognize a key property: In each of the ranges between the roots of $p, q$, the signs of both $p$ and $q$ do not change. Since the ranges cover all roots of $p, q$, the truth value of the formula at a single point in a range is representative of the truth value of the formula on the entire range. To discretize the QE problem, we need only pick one sample point for each range.

However, we want to pick appropriate sample points for any possible $p$ and $q$. The points we pick as represen-

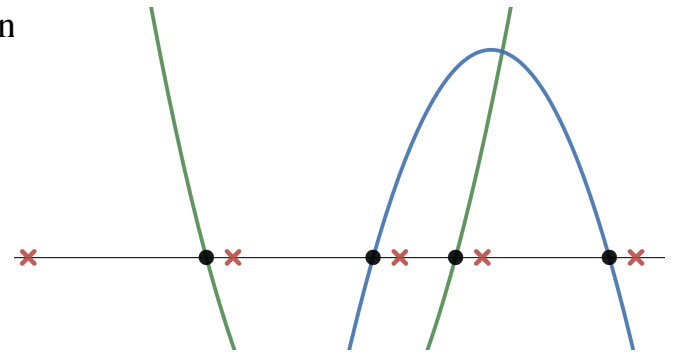

Figure 1: Two quadratics, their roots (black dots) and off-roots (red x's) tatives are called the off-roots, which occur $\epsilon$ units away from the roots, where $\epsilon>0$ is arbitrarily small. We additionally need a representative for the leftmost range, which we represent with the point $-\infty$, where $-\infty$ is arbitrarily negative. Of course, we cannot directly substitute $\epsilon$ and $-\infty$ : they are not real numbers! However, we can virtually substitute them.

\subsubsection{Negative Infinity.}

Given any formula $F$, the VS of $-\infty$ should satisfy the equivalence $F_{x}^{-\infty} \longleftrightarrow \exists y . \forall x<y . F(x)$ (where $y$ does not occur in $F$ ). Intuitively, this says that $-\infty$ acts as if it is arbitrarily negative (so less than the $x$ component of all roots of the polynomials in $F$ ) and captures information for the leftmost range on the real number line in any valuation of the non- $x$ variables. If formula $\exists y . \forall x<y . a x^{2}+b x+c=0$ is true, where $a, b, c$ are polynomials that do not involve $x$, then $a x^{2}+b x+c=0$ holds at infinitely many $x$; since nonzero polynomials have finitely many roots, this can only happen if $a x^{2}+b x+c$ is the zero polynomial in $x$, i.e., it holds that:

$$
\left(a x^{2}+b x+c=0\right)_{x}^{-\infty} \longleftrightarrow a=0 \wedge b=0 \wedge c=0
$$

The negation of (2) captures the behavior of $\neq$ atoms. For $<$ atoms, note that the sign value at $-\infty$ is dominated by the leading coefficient, so:

$$
\left(a x^{2}+b x+c<0\right)_{x}^{-\infty} \longleftrightarrow a<0 \vee(a=0 \wedge(b>0 \vee(b=0 \wedge c<0)))
$$


Finally, $\left(a x^{2}+b x+c \leq 0\right)_{x}^{-\infty} \longleftrightarrow\left(a x^{2}+b x+c=0\right)_{x}^{-\infty} \vee\left(a x^{2}+b x+c<0\right)_{x}^{-\infty}$

In Isabelle/HOL, we formalize that our virtual substitution of $-\infty$ satisfies the desired equivalence (on $\mathbb{R}$ using Remark 1 in in the following lemma:

lemma infinity_evaluni: shows " $\exists y \cdot \forall x<y$. aEvaluni At $x)=$ (evalUni (substNegInfinityUni At) $x$ ) "

To explain this lemma, we need to take a slight detour and discuss a few structural details of our framework (which is discussed in greater detail in Sect.4). The datatype atomUni contains a triple of real numbers (which represent the coefficients of a univariate quadratic polynomial) and a sign condition:

datatype atomUni = LessUni "real*real*real" / EqUni "real*real*real"

l LeqUni "real*real*real" | NeqUni "real*real*real"

The aEvalUni function has type atomUni $\Rightarrow$ real $\Rightarrow$ bool; that is, it takes a sign condition with a triple of real numbers $(a, b, c)$ and a real number $x$ and evaluates whether $a x^{2}+b x+c$ satisfies the sign condition. The evalUni function has type atomUni fmUni $\Rightarrow$ real $\Rightarrow$ bool, where an atomUni fmUni is a formula that involves conjunctions and disjunctions of elements of type atomUni (and "True" and "False"). That is, the evalUni function takes such a formula and a real number and evaluates whether the formula is true at the real number. Thus, infinity_evalUni states that, given At of type atomUni, with tuple $(a, b, c)$ and sign condition $\sim \in\{<,=, \leq, \neq\}, A t_{x}^{-\infty}$ holds iff $\exists y . \forall x<y . a x^{2}+b x+c \sim 0$. This captures the desired equivalence.

Note that these definitions are set up for univariate polynomials (coefficients are assumed to be real numbers). This is deliberate. In Sect.4.3, we will discuss how our framework reduces correctness results for multivariate polynomials to univariate lemmas like infinity_evalUni.

\subsubsection{Infinitesimals.}

Given arbitrary $r$ (not containing $x$ ), VS for $r+\epsilon$ for variable $x$ should capture the equivalence $F_{x}^{r+\epsilon} \longleftrightarrow$ $\exists y>r . \forall x \in(r, y] . F(x)$, where $F$ does not contain $y$. Intuitively, this says that (in any valuation of the non- $x$ variables) $r+\epsilon$ captures information for the interval between $r$ and the next greatest $x$-root.

For $=$ and $\neq$ atoms, we proceed in the same manner as we did with $-\infty$, as $(r, y]$ contains infinitely many points and only the zero polynomial has infinitely many solutions. As before, $\leq$ atoms turn into disjunctions of the inequality and equality representations at $r+\epsilon$. We are left only to consider $<$ atoms.

Consider $(p<0)_{x}^{r+\epsilon}$ where $p=a x^{2}+b x+c$ with polynomials $a, b, c$ not containing $x$, and an arbitrary $r$ not containing $x$. Notice that if $(p<0)_{x}^{r}$, then because polynomials are continuous, we can choose a small enough $y$ so that $\forall x \in(r, y] . p<0$. If instead $(p=0)_{x}^{r}$, then consider the partial derivative of $p$ evaluated at $r$. If $\frac{\partial p}{\partial x}(r)$ is negative, then $\exists y>r . \forall x \in(r, y] . p<0$ holds, because $p$ is decreasing in $x$ locally after $x=r$. If $\frac{\partial p}{\partial x}(r)$ is positive, then $\exists y>r . \forall x \in(r, y] . p<0$ does not hold, because $p$ is increasing in $x$ after $x=r$. If $\frac{\partial p}{\partial x}(r)$ is zero, then to ascertain whether $\exists y>r . \forall x \in(r, y] . p<0$, we will need to check higher derivatives.

This pattern forms the following recurrence, with the base case $(p<0)_{x}^{r+\epsilon}=(p<0)_{x}^{r}$ for polynomials $p$ of degree zero:

$$
(p<0)_{x}^{r+\epsilon} \stackrel{\text { def }}{=}(p<0)_{x}^{r} \vee\left((p=0)_{x}^{r} \wedge((\partial p / \partial x)<0)_{x}^{r+\epsilon}\right)
$$

We use the VS algorithm from Section 3.2 to characterize $(p<0)_{x}^{r}$ and $(p=0)_{x}^{r}$.

In Isabelle/HOL, we show that given a quadratic root $r$, the virtual substitution of $r+\epsilon$ satisfies the desired equivalence in the following theorem (on $\mathbb{R}$ using Remark 1 ; we have an analogous lemma for linear roots $r$ ): 


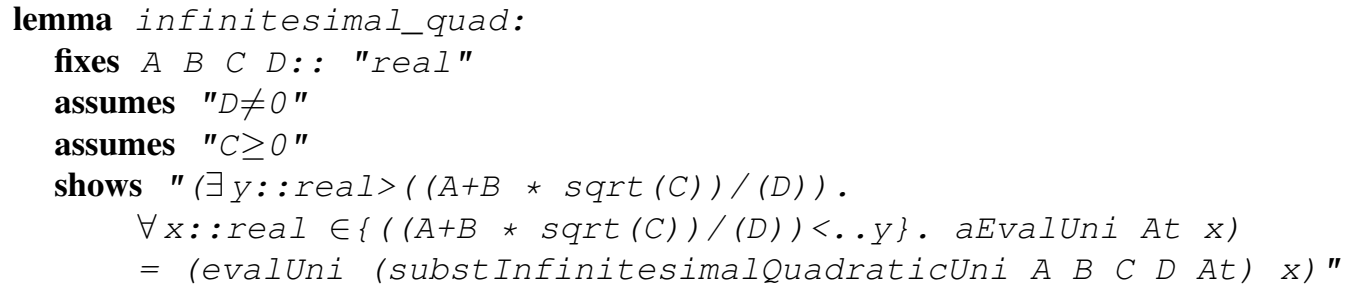

Note that $\{r<\ldots y\}$ in Isabelle stands for the range $(r, y]$. This says that, given At of type atomUni, with tuple $(a, b, c)$ and sign condition $\sim \in\{<,=, \leq, \neq\}, A t_{x}^{r+\epsilon}$ holds iff $\exists y>r . \forall x \in(r, y] . a x^{2}+b x+c \sim 0$, which is the desired equivalence.

\subsubsection{The General VS Theorem.}

Now that we have explained virtually substituting $-\infty$ and infinitesimals, we are ready to state the general VS theorem.

Let $F$ be a formula of the following shape, where each $a_{i}, b_{i}, c_{i}$, and $d_{i}$ is a polynomial that is at most quadratic in variable $x$ :

$$
F=\left(\bigwedge a_{i}=0\right) \wedge\left(\bigwedge b_{i}<0\right) \wedge\left(\bigwedge c_{i} \leq 0\right) \wedge\left(\bigwedge d_{i} \neq 0\right)
$$

Let $R(p)$ denote the set of symbolic expressions of the form $\left(g_{1}+g_{2} \sqrt{g_{3}}\right) / g_{4}$ that, as in Sect. 3.2, are roots of the polynomial $p$ in $x$, where the $g_{i}$ 's are polynomials not involving $x$. For the zero polynomial, let $R(0)=\emptyset$. Note that, as in Sect. 3.2, the $g_{i}$ 's come with certain well-definedness checks that we retain implicitly in the construction (for example, $g_{4} \neq 0$ and $g_{3} \geq 0$ ). We now define:

$$
A=\bigcup R\left(a_{i}\right) \quad B=\bigcup R\left(b_{i}\right) \quad C=\bigcup R\left(c_{i}\right) \quad D=\bigcup R\left(d_{i}\right)
$$

Then we obtain the following QE equivalence, where for simplicity we elide the relevant crucial welldefinedness checks (cross-reference [20, Theorem 21.1]):

$$
(\exists x . F) \longleftrightarrow F_{x}^{-\infty} \vee \bigvee_{r \in A \cup C} F_{x}^{r} \vee \bigvee_{r \in B \cup C \cup D} F_{x}^{r+\epsilon}
$$

Intuitively, this formula states that if there is a particular $x$ that satisfies $F$, then it must be the case that $x$ is one of the equality roots from $A \cup C$, or that $x$ falls in one of the particular ranges (including $-\infty$ as a range) obtained by partitioning the number line by the roots in $B \cup C \cup D$.

Equation (3) can be optimized further by eliding $C$ from the off-roots:

$$
(\exists x . F) \longleftrightarrow F^{-\infty} \vee \bigvee_{r \in A \cup C} F_{x}^{r} \vee \bigvee_{r \in B \cup D} F_{x}^{r+\epsilon} .
$$

Intuitively, this optimization holds because polynomials are continuous. More precisely, if $F$ has the shape $F=(p \leq 0 \wedge G)$, and if $r$ is an $x$-root of $p$, then $r$ already satisfies $p \leq 0$ in any valuation of the non- $x$ variables, so including $r+\epsilon$ as a sample point on account of $p \leq 0$ is redundant. It is possible that $G$ contains some atom $q<0$ or $q \neq 0$ where $r$ is an $x$-root of $q$. In this case, $r+\epsilon$ will already be a sample point on account of $q$, and we do not need to add it in on account of $p$. Alternatively, if $G$ does not contain such a $q$, then, in any valuation of the non- $x$ variables, it is impossible for $G$ to be satisfied by $r+\epsilon$ and not $r$, meaning that it is redundant to include $r+\epsilon$ as a sample point on account of $G$.

The general QE theorem is proved in Isabelle/HOL as the following, using Remark 1 to restrict to the univariate case and avoid well-definedness formulas: 


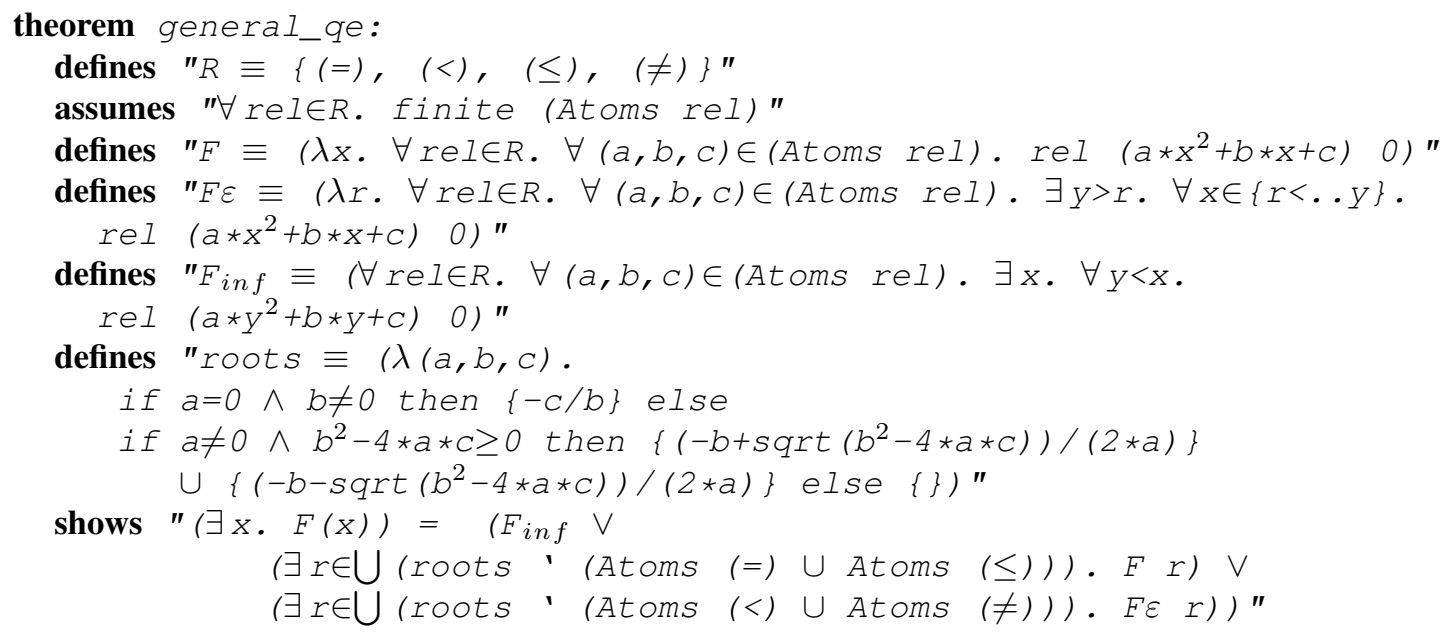

Here, ' is the Isabelle/HOL syntax for mapping a function over a set. This theorem says that if a finitelength formula $F$ is of the requisite shape, then there exists an $x$ satisfying $F$ iff $F$ is satisfied at $-\infty$ (captured by $F_{\text {inf }}$ ), or there is a root $r$ of one of the $=$ or $\leq$ atoms where $F r$ holds, or if there is a root $r$ of one of the $<$ or $\neq$ atoms where $F_{\varepsilon} r$ holds. The proof is quite lengthy and involves a significant amount of casework; however, because we are working with univariate polynomials thanks to Remark1, this casework mostly reduces to arithmetic computations and basic real analysis for univariate polynomials, and some of what we need, such as properties of discriminants and continuity properties of polynomials, is already formalized in Isabelle/HOL's standard library.

\subsection{Top Level Algorithms}

We develop several top-level algorithms that perform these VS procedures on multivariate polynomials; these are described in more detail in Appendix B. Crucially, each features its own proof of correctness. For example, for the VSEquality algorithm, which performs equality VS repeatedly, we have:

theorem VSEquality_eval: " $\forall \nu$. eval (VSEquality $\varphi$ ) $\nu=$ eval $\varphi \nu "$

Here, the eval function expresses the truth value of the (multivariate) input formula given a valuation $x S$, represented as a list of real numbers. Since we quantify over all possible valuations and express that they are the same before and after running the algorithm, we prove the soundness of VSEquality. The correctness of this theorem only relies on Isabelle/HOL's trusted core.

As our algorithms are general enough to handle formulas with high degree polynomials where VS does not apply, we cannot assert that the result is quantifier free (it might not be). To demonstrate the practical usefulness of these algorithms, we export our code to SML and experimentally show that these algorithms solve many benchmarks. The code exports rely on the correctness of Isabelle/HOL's code export, which ongoing work is attempting to establish [12].

\section{Framework}

We turn to a discussion of our framework, which is designed with two key goals in mind: First, perform VS as many times as possible on any given formula. Second, reduce unwieldy multivariate proofs to more manageable univariate ones. 


\subsection{Representation of Formulas}

We define our type for formulas in the canonical datatype $\mathrm{fm}$ :

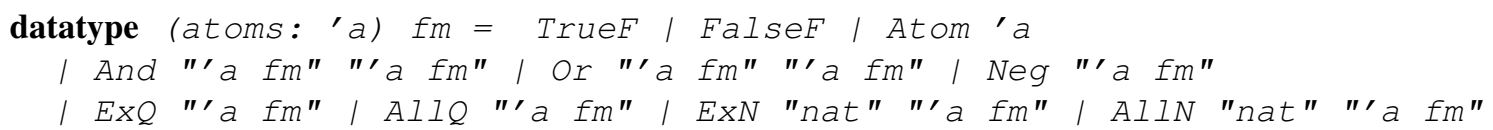

As in Nipkow's previous work [17], we use De Bruijn indices to express the variables: That is, the 0th variable represents the innermost quantifier, and variables greater than the number of quantifiers represent the free variables.

We have two constructors for each type of quantifier: $E \times Q F$ (resp. AllQ $F$ ) indicates a single existential (universal) quantifier, and $E x N \cap \cap$ (resp. AlIN $\cap F$ ) represents a block of $n$ existential (universal) quantifiers. These representations are interchangeable and converted back and forth in our algorithm; we include the block representation for variable ordering heuristics (see Appendix C.3).

We utilize the multivariate polynomial library [26] to define our atoms:

datatype atom = Less "real mpoly" / Eq "real mpoly" / Leq "real mpoly"

I Neq "real mpoly"

Each atom is normalized without loss of generality, so that the atom Less $p$ means $p<0, E q p$ means $p=0$, and so on.

For example, the $\mathrm{FOL}_{\mathbb{R}}$ formula $\forall x .\left(\left(\exists y . x a=y^{2} b\right) \wedge \neg\left(\forall y .5 x^{2} \leq y\right)\right)$ is represented in our framework as follows, where Const $n$ represents the constant $n \in \mathbb{R}$, and $\operatorname{Var} i$ represents the $i$ th variable:

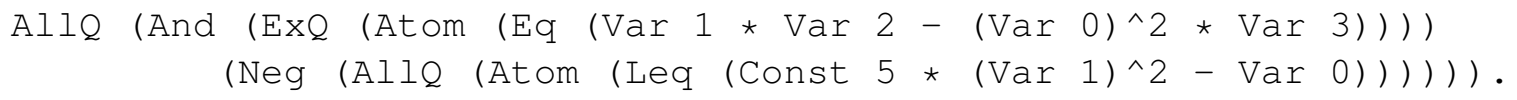

Note that we could restrict ourselves to the $\top, \neg, \vee, \exists$ connectives and normalize $\leq$ and $\neq$ atoms to combinations of $<$ and $=$ atoms, and we could still express all of $\mathrm{FOL}_{\mathbb{R}}$. We avoid this for two reasons: because it would linearly increase the size of the formula, and because we want to handle $\leq$ atoms in the optimized way discussed in Sect.3.3.3 (see (4)). We do, however, allow for the normalization of $p=q$ into $p-q=0$. This does not affect the size of the formula, and can afford simplifications: For example, $x^{3}+x^{2}+x+1=x^{3}$ becomes $x^{2}+x+1=0$.

\subsection{Modified Disjunctive Normal Form}

Nipkow's prior work [17] avoided incurring cases where linear VS does not apply by constraining atoms to be linear. In order to develop a general-purpose VS method which can be used, e.g., as a preprocessing method for CAD, we must reason about cases where VS fails to perform QE for a specific quantifier, and still continue the execution of the algorithm to the remaining quantifiers to simplify the formula as much as possible. To help with this, we implement a modified disjunctive normal form (DNF) that allows expressions to involve quantifiers.

\subsubsection{Contextual Awareness.}

Let us analyze how to increase the informational content in a formula with respect to a quantified variable of interest.

Say we wish to perform VS to eliminate variable $x$ in the formula $\exists x . F$, where $F$ is not necessarily quantifier free. In linear time, we remove all negations from the formula by converting it into negation 
normal form. We can then normalize $\exists x . F$ into the following form, where the $A_{n, i}$ 's are (quantifier-free) atoms:

$$
\exists x \cdot \bigvee_{n}\left(\bigwedge_{i} A_{n, i} \wedge \bigwedge_{j}\left(\forall y \cdot F_{n, j}\right) \wedge \bigwedge_{k}\left(\exists z \cdot F_{n, k}\right)\right) .
$$

This normalization procedure is similar to standard DNF, as it handles quantified formulas as if they were atomic formulas. We can distribute the existential quantifier across the disjuncts, which results in the equivalent formula:

$$
\bigvee_{n} \exists x \cdot\left(\bigwedge_{i} A_{n, i} \wedge \bigwedge_{j}\left(\forall y \cdot F_{n, j}\right) \wedge \bigwedge_{k}\left(\exists z \cdot F_{n, k}\right)\right) .
$$

Now we run the VS algorithm, i.e. the input to VS is a conjunction of atomic formulas and quantified formulas in the shape of (5). Notice that if equality VS applies to atom $A_{n, i}$, then the relevant roots can be substituted into the quantified formulas $F_{n, j}$ and $F_{n, k}$, but roots from $F_{n, j}$ or $F_{n, k}$ cannot be substituted into $A_{n, i}$ since they feature quantified variables which are undefined in the broader context. So, our informational content is greatest when the number of $A_{n, i}$ atoms is maximized and the sizes of the $F_{n, j}$ and $F_{n, k}$ are minimized.

\subsubsection{Innermost Quantifier Elimination.}

The innermost quantifier has an associated formula which is entirely quantifier free (and thus has no $F_{n, j}$ and $F_{n, k}$ ). As such, we opt to perform VS recursively, starting with the innermost quantifier and moving outwards, hoping that VS is successful and the quantifier-free property is maintained. This is not always optimal. Consider the following formula:

$$
\exists x .\left(x=0 \wedge \exists y \cdot x y^{3}+y=0\right) .
$$

If we attempt to perform quadratic VS on the innermost $y$ quantifier, it is cubic and will fail. However, performing VS on the $x$ quantifier first fixes $x=0$, which converts the cubic $x y^{3}+y=0$ equality into the linear $y=0$. So, an (unoptimized) run of inside-out VS would produce $\exists y . y=0$, and we could completely resolve the QE query by running VS again.

\subsubsection{Reaching Under Quantifiers.}

We would like to recover usable information from the $F_{n, k}$ formulas to increase the informational content going into our QE algorithm. It would be ideal if we could "reach underneath" the existential binders and "pull out" the atoms from the formulas. We can achieve this through a series of transformations. Let $k$ range from 0 to $K_{n}$. If we pull out each existential quantifier one by one, we get the following formula, which is equivalent to formula (5):

$$
\bigvee_{n} \exists z_{0} \cdot \cdots \exists z_{K_{n}} \cdot \exists x \cdot\left(\bigwedge_{i} A_{n, i} \wedge \bigwedge_{j}\left(\forall y \cdot F_{n, j}\right) \wedge \bigwedge_{k} F_{n, k}\right)
$$

This works because the rest of the conjuncts do not mention the quantified variable $z_{k}$ and adjacent existential quantifiers can be swapped freely (without changing the logical meaning of the formula).

We can then recursively unravel the formulas $F_{n, k}$, moving as many existential quantifiers as possible to the front. Our implementation does this via a bottom-up procedure, starting underneath the innermost existential quantifier and building upwards, normalizing the formula into the form:

$$
\bigvee_{n} \exists z_{0} \cdot \cdots \exists z_{K_{n}} \cdot \exists x \cdot\left(\bigwedge_{i} A_{n, i} \wedge \bigwedge_{j} \forall y \cdot F_{n, j}\right)
$$


On paper, these transformations are simple as they involve named quantified variables; however, because our implementation uses a locally nameless form for quantifiers with de Bruijn indices, shifting an existential quantifier requires a "lifting" procedure $A \uparrow$ which increments all the variable indices in $A$ by one. This allows for the following conversion: $A \wedge \exists z \cdot F \longleftrightarrow \exists z \cdot((A \uparrow) \wedge F)$.

\subsection{Logical Evaluation}

Our proofs show that the input formula and the output formula (after VS) are logically equivalent, i.e., have the same truth value under any valuation. This needs a method of "plugging in" the real-valued valuation into the variables of the polynomials. Towards this, we define the eval function, which accumulates new values into the valuation as we go underneath quantifiers, and the aEval function, which homomorphically evaluates a polynomial at a valuation.

When proving correctness, we focus our attention on one quantifier at a time. By Remark1, correctness of general VS follows when considering a formula $F$ with a single quantifier, where $F$ contains only polynomials of at most degree two (otherwise general VS does not apply). With these restrictions, we can substitute a valuation into the non-quantified variables, transforming multivariate polynomials into univariate polynomials. For example, let $a, b$, and $c$ be arbitrary multivariate polynomials that do not mention variable $x$. Let $\hat{p}=\gamma(p)$ denote the evaluation of polynomial $p$ at valuation $\gamma$ ( $\hat{p}$ is a real number). We obtain the following conversion between multivariate and univariate polynomials:

$$
\text { eval }\left(a x^{2}+b x+c=0\right) \gamma \longleftrightarrow \text { evalUni }\left(\hat{a} x^{2}+\hat{b} x+\hat{c}=0\right) \hat{x}
$$

As such, we develop an alternative VS algorithm for univariate polynomials, where atoms are represented as triples of real-valued coefficients (as seen in Sect. 3.3), and show that under this specific valuation, the multivariate output is equivalent per valuation with the output of the univariate case. Thus, we finish the proof of the multivariate case by lifting the proof for the univariate case.

\subsection{Polynomial Contributions}

We build on the polynomials library [26], which was designed to support executable multivariate polynomial operations. This choice naturally comes with tradeoffs, and a number of functions and lemmas that we needed were missing from the library. For example, we needed an efficient way to isolate the coefficient of a variable within a polynomial, which we define in the isolate_variable_sparse function. The following particularly critical lemma rewrites a multivariate polynomial in $\mathbb{R}\left[a_{1}, \ldots, a_{n}, x\right]$ as a nested polynomial $\mathbb{R}\left[a_{1}, \ldots, a_{n}\right][x]$, i.e., a univariate polynomial in $\mathrm{x}$ with coefficients that are polynomials in $\mathbb{R}\left[a_{1}, \ldots, a_{n}\right]$ :

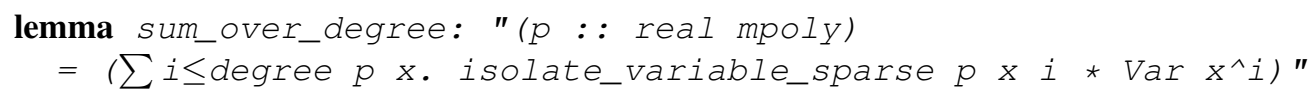

This is needed rather frequently within VS, as we often seek to re-express polynomials with respect to a single quantified variable of interest, and although it is mathematically quite obvious, its verification was somewhat involved.

Additionally, to utilize the variables within polynomials as de Bruijn indices, we implemented various lifting and substitution operations. These include the liftPoly and lowerPoly variable reindexing functions. These and other contributions to the polynomials library are discussed in Appendix B.4. 


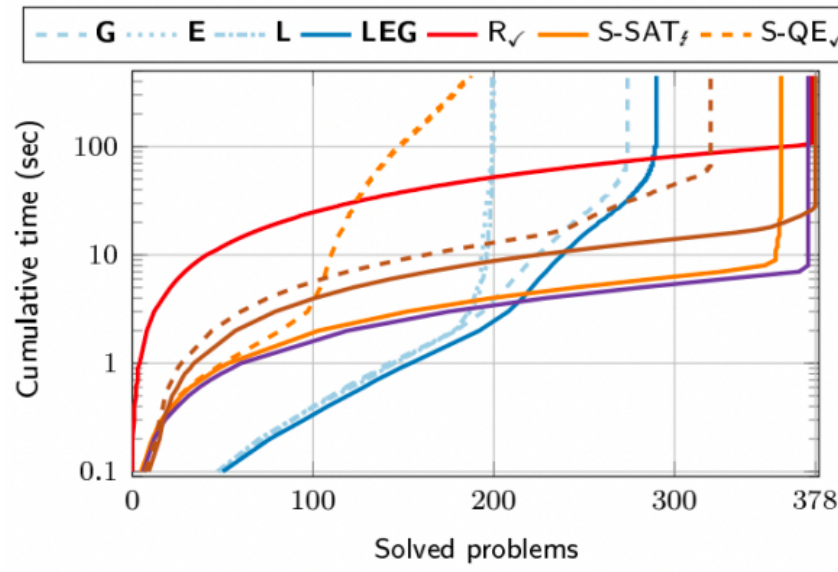

(a) CADE09 (378 examples)

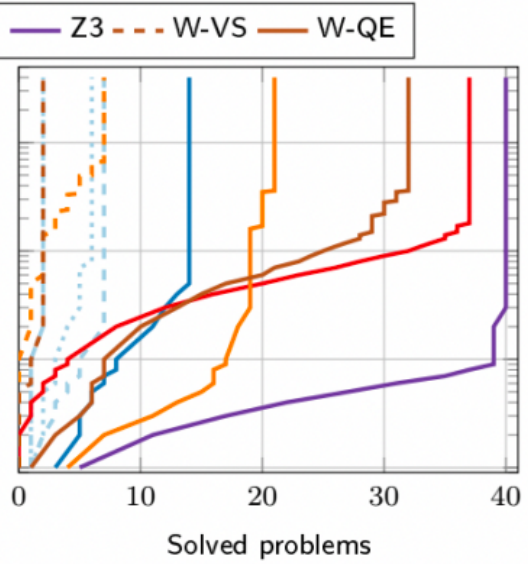

(b) Economics (45 examples)

Figure 2: Cumulative time to solve fastest $n$ problems (flatter and more is better)

\section{Experiments}

The benchmark suite consists of 378 QE problems in category CADE09 collected from 94 examples [21], and category Economics with 45 QE problems [16]. CADE09 and Economics examples were converted into decision problems, powers were flattened to multiplications, and CADE09 were additionally rewritten to avoid polynomial division. For sanity checking, we also negated the CADE09 examples [21]. We run on commodity hardware $\left.\right|^{2}$ The benchmark examples, as well as all scripts to rerun the experiments are in [24].

Tools. We compare the performance of $a$ ) our VSEquality (E), VSGeneral $(\mathbf{G})$, VSLucky (L), and VSLEG (LEG) algorithms (Appendix B) to $b$ ) Redlog [7] snapshots 2021-04-13 $]^{3}\left(\mathbf{R}_{\xi}\right)$ and 2021-07-16 $\left(\mathbf{R}_{\checkmark}\right.$, which includes bug fixes for contradictions we reported to Redlog developers), c) SMT-RAT 21.05 [5] quantifier elimination $\left(\mathbf{S}-\mathbf{Q E}_{\checkmark}\right)$ and satisfiability checking $\left(\mathbf{S}_{\mathbf{S}} \mathbf{S A T} \mathbf{T}_{\xi}\right), d$ ) the SMT solver Z3 4.8.10 [6] (Z3), and e) Wolfram Engine 12.3.1(W-VS, W-QE). All tools were run in Docker containers on Ubuntu 18.04 with $8 \mathrm{~GB}$ of memory and $6 \mathrm{CPU}$ cores. Tool syntax translations from SMT-LIB format were done prior to benchmarking: For our VS algorithms, examples were translated to SML data structures and compiled with MLton7; as a result, measurements do not include parsing. For W-VS and W-QE, examples were translated into Wolfram syntax, including configuration options restricting QE to quadratic virtual substitution in WVS. For S-QE ${ }_{\checkmark}$, check-sat was replaced with eliminate-quantifiers.

Results. Each example has a timeout of 30s. Figure 2 summarizes the performance on the CADE09 and Economics examples in terms of the cumulative time needed to solve (return "true", "false", "sat", or "unsat") the fastest $n$ problems with a logarithmic time axis: more problems solved and a flatter curve is better.

\footnotetext{
${ }^{2}$ MacBook Pro 2019 with 2.6GHz Intel Core i7 (model 9750H) and 32GB memory (2667MHz DDR4 SDRAM).

3 https://sourceforge.net/projects/reduce-algebra/files/snapshot_2021-04-13/

4 https://sourceforge.net/projects/reduce-algebra/files/snapshot_2021-07-16/

5 https://qithub.com/ths-rwth/smtrat/releases/taq/21.05

https://qithub.com/Z3Prover/z3/releases/tag/z3-4.8.10

http://mlton.org/
} 


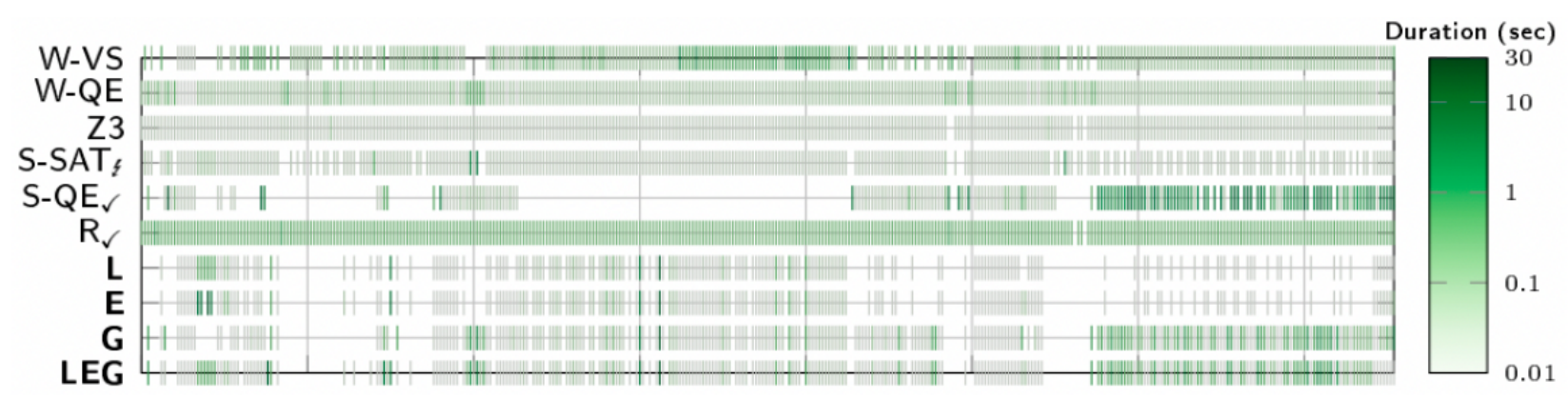

Figure 3: CADE09 duration per problem (color indicates duration, lighter is better)

Wolfram Engine solves all problems in the CADE09 category, closely trailed by Redlog, Z3. The near constant computation time offset of Redlog in comparison to Z3, SMT-RAT, and Wolfram Engine may be attributable to the additional step of entering an SMT REPL. Our verified VSEquality (E), VSGeneral (G), VSLucky (L), and VSLEG (LEG) algorithms rank in performance between the basic quantifier elimination implementation in SMT-RAT ( $\mathrm{S}-\mathrm{QE}_{\checkmark}$ ), virtual substitution in Wolfram Engine (W-VS), full SMT approaches $\left(\mathrm{S}_{-} \mathrm{SAT}_{\hat{\xi}}, \mathrm{Z} 3\right)$, and combined virtual substitution plus $\mathrm{CAD}$ implementations $\left(\mathrm{R}_{\checkmark}, \mathrm{W}-\mathrm{QE}\right)$. The reduced startup time of our algorithms is attributable to the omitted parsing step. Overall, VSEquality and VSLucky solve examples fast, but the wider applicability of VSGeneral and VSLEG allows them to solve considerably more examples. Though we have already implemented a number of optimizations for VS (Appendix C we do not expect to outperform prior tools at this stage, as many of them have been optimized over a period of many years.

A comparison of duration per problem is in Fig. 3. Though there is considerable overlap between VSEquality, VSGeneral, and VSLucky, mutually exclusive sets of solved examples (and considerable performance differences on a number of examples) foreshadow the performance achievable with the combined VSLEG algorithm.

Contradictions. In Fig. 4, we compare the CADE09 results to the results on negated CADE09 examples to highlight contradictions between answers (e.g., both $A$ and $\neg A$ are claimed to be true). Wolfram Engine and Z3 answer consistently on both formula sets, and solve (almost) all examples. Redlog, the main VS implementation, in $\mathrm{R}_{\varepsilon}$ and previous versions in general does not perform well on the negated formulas and reports 96 contradictory answers; the contradictory examples were shared with the developers and triggered several bug fixes that are now available in $\mathrm{R}_{\checkmark}$ (no contradictions found on the benchmark set). SMTRAT performs better than $\mathrm{R}_{\xi}$ on the negated formulas, but in satisfiability mode contradicts itself on 41 examples by silently ignoring quantifiers in the input; in quantifier elimination mode, SMT-RAT supports quantifiers and does not report contradictions, but SMT-RAT then incurs a significant performance loss

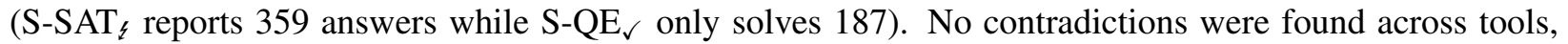
i.e., whenever a tool's answers were consistent internally, the answers agreed with those of other tools. Our VSLEG algorithm has similar performance for proving and disproving in terms of absolute number of solved examples, but combining proving and disproving would still solve more examples than just one question individually (as for $\mathrm{S}-\mathrm{QE}_{\checkmark}$ and W-VS).

In summary, the performance of our verified virtual substitution $Q E$ on the benchmark set is encouraging. The number of solved examples is close to other VS implementations (304 examples by our VSLEG vs. 322 by $\mathrm{W}-\mathrm{VS}$ ) and the cumulative solving time reveals that the majority of examples are solved fast. 


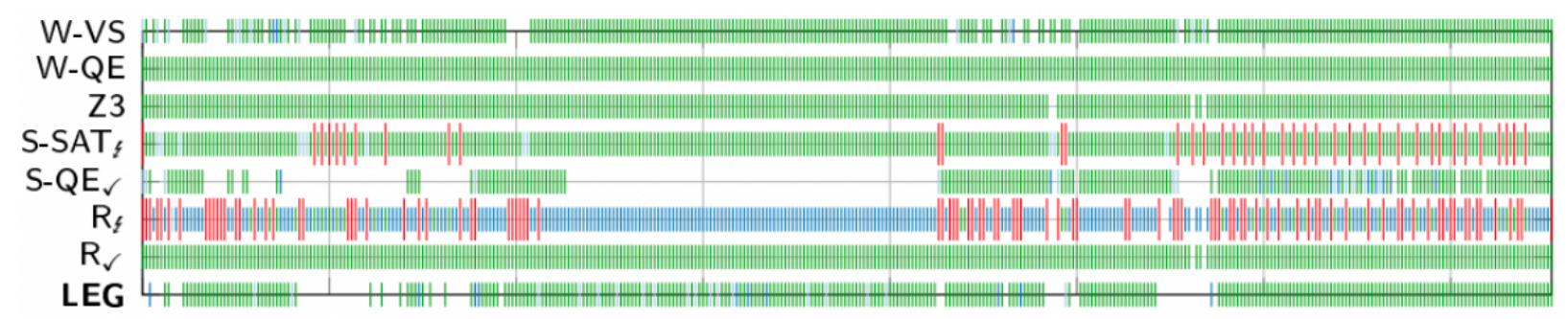

Figure 4: CADE09 consistency comparison between original and negated formula: color indicates dis-

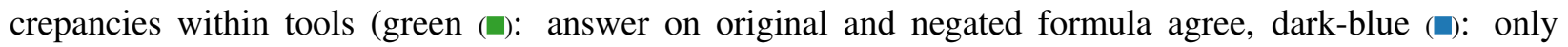
original solved, light-blue $(\square)$ : only negated solved, red+long $(\square)$ : contradictory answers (both formulas unsat/proved or both sat/disproved), empty: both timeout/unknown)

\section{Conclusion and Future Work}

We verify linear and quadratic virtual substitution for real arithmetic; our algorithms are provably correct up to Isabelle/HOL's trusted core and code export. Developing practical verified VS in Isabelle/HOL required significant low-level improvements and extensions to Isabelle's multivariate polynomials library. Our extensive experiments both reveal the benefits of our current optimizations and indicate room for future improvements. Further optimizations to the polynomial libraries, such as efficient coefficient lookup for polynomials using red black trees, would be welcome. Expanding our framework to handle formulas that involve polynomial division would also be of practical significance. Continuing to develop our formalization with such improvements is of especial significance given that our experiments found long-standing errors in existing unverified real arithmetic tools. This demonstrates that, even if verification were not a virtue in and of itself, real arithmetic is so subtle that formal verification is the best way toward an implementation that is both useful and correct in practice.

Acknowledgments. We wish to thank Fabian Immler for his substantial contributions at CMU to the polynomial theories of Isabelle/HOL and regret that his current industry position precludes our ability to include him as a coauthor. Thank you also to the anonymous FM reviewers for their useful feedback.

This material is based upon work supported by the National Science Foundation under Grant No. CNS1739629, a National Science Foundation Graduate Research Fellowship under Grants Nos. DGE1252522 and DGE1745016, and by the AFOSR under grant number FA9550-16-1-0288. Any opinions, findings, and conclusions or recommendations expressed in this material are those of the author(s) and do not necessarily reflect the views of the National Science Foundation or of AFOSR.

\section{References}

[1] Brandon Bohrer, Vincent Rahli, Ivana Vukotic, Marcus Völp, and André Platzer. Formally verified differential dynamic logic. In Yves Bertot and Viktor Vafeiadis, editors, CPP, pages 208-221, New York, 2017. ACM. doi:10.1145/3018610.3018616.

[2] Amine Chaieb. Automated methods for formal proofs in simple arithmetics and algebra. $\mathrm{PhD}$ thesis, Technische Universität München, 2008. URL: https://mediatum.ub.tum.de/doc/ $649541 / 649541 \cdot \mathrm{pdf}$. 
[3] Cyril Cohen and Assia Mahboubi. Formal proofs in real algebraic geometry: from ordered fields to quantifier elimination. Log. Methods Comput. Sci., 8(1), 2012. doi:10.2168/LMCS-8 (1: 2) 2012 .

[4] George E. Collins. Quantifier elimination for real closed fields by cylindrical algebraic decomposition. In H. Barkhage, editor, Automata Theory and Formal Languages, volume 33 of LNCS, pages 134-183. Springer, 1975. doi:10.1007/3-540-07407-4\_17.

[5] Florian Corzilius, Gereon Kremer, Sebastian Junges, Stefan Schupp, and Erika Ábrahám. SMTRAT: an open source C++ toolbox for strategic and parallel SMT solving. In Marijn Heule and Sean A. Weaver, editors, SAT, volume 9340 of LNCS, pages 360-368. Springer, 2015. doi: 10.1007/978-3-319-24318-4\_26.

[6] Leonardo Mendonça de Moura and Nikolaj Bjørner. Z3: an efficient SMT solver. In C. R. Ramakrishnan and Jakob Rehof, editors, TACAS, volume 4963 of LNCS, pages 337-340. Springer, 2008. doi:10.1007/978-3-540-78800-3\_24.

[7] Andreas Dolzmann and Thomas Sturm. REDLOG: computer algebra meets computer logic. SIGSAM Bull., 31(2):2-9, 1997. doi:10.1145/261320.261324.

[8] Antonio J. Durán, Mario Pérez, and Juan L. Varona. The misfortunes of a trio of mathematicians using computer algebra systems. can we trust in them? Notices of the AMS, 61(10):1249-1252, 2014. doi:10.1090/noti1173.

[9] Dorian Florescu and Matthew England. Algorithmically generating new algebraic features of polynomial systems for machine learning. CoRR, abs/1906.01455, 2019. URL: http://arxiv.org/ abs/1906.01455, arXiv:1906.01455.

[10] Nathan Fulton, Stefan Mitsch, Jan-David Quesel, Marcus Völp, and André Platzer. KeYmaera $\mathrm{X}$ : An axiomatic tactical theorem prover for hybrid systems. In Amy P. Felty and Aart Middeldorp, editors, CADE, volume 9195 of $L N C S$, pages 527-538. Springer, 2015. doi:10.1007/ 978-3-319-21401-6\_36.

[11] Sicun Gao, Soonho Kong, and Edmund M. Clarke. dReal: An SMT solver for nonlinear theories over the reals. In Maria Paola Bonacina, editor, CADE, volume 7898 of LNCS, pages 208-214. Springer, 2013. doi:10.1007/978-3-642-38574-2\_14.

[12] Lars Hupel and Tobias Nipkow. A verified compiler from Isabelle/HOL to CakeML. In Amal Ahmed, editor, ESOP, volume 10801 of $L N C S$, pages 999-1026. Springer, 2018. doi:10.1007/ 978-3-319-89884-1\_35.

[13] Dejan Jovanovic and Leonardo Mendonça de Moura. Solving non-linear arithmetic. In Bernhard Gramlich, Dale Miller, and Uli Sattler, editors, IJCAR, volume 7364 of LNCS, pages 339-354. Springer, 2012. doi:10.1007/978-3-642-31365-3\_27.

[14] Marek Košta. New concepts for real quantifier elimination by virtual substitution. PhD thesis, Universität des Saarlandes, 2016.

[15] Sean McLaughlin and John Harrison. A proof-producing decision procedure for real arithmetic. In Robert Nieuwenhuis, editor, CADE, volume 3632 of $L N C S$, pages 295-314. Springer, 2005. doi: $10.1007 / 11532231 \backslash \_22$.

[16] Casey B. Mulligan, Russell J. Bradford, James H. Davenport, Matthew England, and Zak Tonks. Quantifier elimination for reasoning in economics. CoRR, abs/1804.10037, 2018. URL: http: //arxiv.org/abs/1804.10037, arXiv:1804.10037, 
[17] Tobias Nipkow. Linear quantifier elimination. J. Autom. Reason., 45(2):189-212, 2010. doi:10. 1007/s10817-010-9183-0.

[18] Grant Olney Passmore. Combined Decision Procedures for Nonlinear Arithmetics, Real and Complex. $\mathrm{PhD}$ thesis, School of Informatics, University of Edinburgh, 2011.

[19] André Platzer. Logical Analysis of Hybrid Systems: Proving Theorems for Complex Dynamics. Springer, Heidelberg, 2010. doi:10.1007/978-3-642-14509-4.

[20] André Platzer. Logical Foundations of Cyber-Physical Systems. Springer, Cham, 2018. doi:10 . 1007/978-3-319-63588-0.

[21] André Platzer, Jan-David Quesel, and Philipp Rümmer. Real world verification. In Renate A. Schmidt, editor, $C A D E$, volume 5663 of $L N C S$, pages 485-501, Berlin, 2009. Springer. doi: $10.1007 / 978-3-642-02959-2 \_35$.

[22] Stefan Ratschan and Jan-Georg Smaus. Verification-integrated falsification of non-deterministic hybrid systems. In Christos G. Cassandras, Alessandro Giua, Carla Seatzu, and Janan Zaytoon, editors, ADHS, volume 39 of IFAC Proceedings Volumes, pages 371-376. Elsevier, 2006. doi:10.3182/ 20060607-3-IT-3902.00068.

[23] Matias Scharager, Katherine Cordwell, Stefan Mitsch, and André Platzer. Verified quadratic virtual substitution for real arithmetic. Archive of Formal Proofs, August 2021. https: / / www . isa-afp. org/entries/Virtual_Substitution.html, Formal proof development.

[24] Matias Scharager, Katherine Cordwell, Stefan Mitsch, and André Platzer. Verified quadratic virtual substitution for real arithmetic: Benchmark examples and scripts. Zenodo, 2021. doi:10.5281/ zenodo.5189881.

[25] A. Seidenberg. A new decision method for elementary algebra. Annals of Math., 60(2):365-374, 1954.

[26] Christian Sternagel and René Thiemann. Executable multivariate polynomials. Archive of Formal Proofs, August 2010. https://www.isa-afp.org/entries/Polynomials.html, Formal proof development.

[27] Thomas Sturm. A survey of some methods for real quantifier elimination, decision, and satisfiability and their applications. Math. Comput. Sci., 11(3-4):483-502, 2017. doi:10.1007/ s11786-017-0319-z,

[28] Thomas Sturm. Thirty years of virtual substitution: Foundations, techniques, applications. In Manuel Kauers, Alexey Ovchinnikov, and Éric Schost, editors, ISSAC, pages 11-16. ACM, 2018. doi: 10 . 1145/3208976.3209030.

[29] Alfred Tarski. A Decision Method for Elementary Algebra and Geometry. RAND Corporation, Santa Monica, CA, 1951.

[30] Volker Weispfenning. The complexity of linear problems in fields. J. Symb. Comput., 5(1/2):3-27, 1988. doi:10.1016/S0747-7171(88)80003-8.

[31] Volker Weispfenning. Quantifier elimination for real algebra - the cubic case. In Malcolm A. H. MacCallum, editor, ISSAC, pages 258-263. ACM, 1994. doi:10.1145/190347.190425.

[32] Volker Weispfenning. Quantifier elimination for real algebra - the quadratic case and beyond. Appl. Algebra Eng. Commun. Comput., 8(2):85-101, 1997. doi:10.1007/s 002000050055. 


\section{A Isabelle/HOL Code}

This appendix contains some of the key Isabelle/HOL code used within the Equality VS algorithm (see Sect. 3.2) and the General VS algorithm (see Sect.3.3.

\section{A.1 Linear Substitution}

In Isabelle/HOL, we formalize linear substitution in the Iinear_substitution function. Here, the call linear_substition $i$ a $b$ A returns the atom $f m$ that is the result of virtually substituting the fraction $a / b$ for variable with de Bruijn index $i$ into atom $A$ (the atom and atom $\mathrm{fm}$ datatypes are explained in Sect.4.1. More precisely, the linear_substition function is taking as input a natural number, which indicates which variable is of interest, two real multivariate polynomials $a$ and $b$, and an atom $A$. It cases on the structure of $A$ and follows the casework described in Sect.3.2.

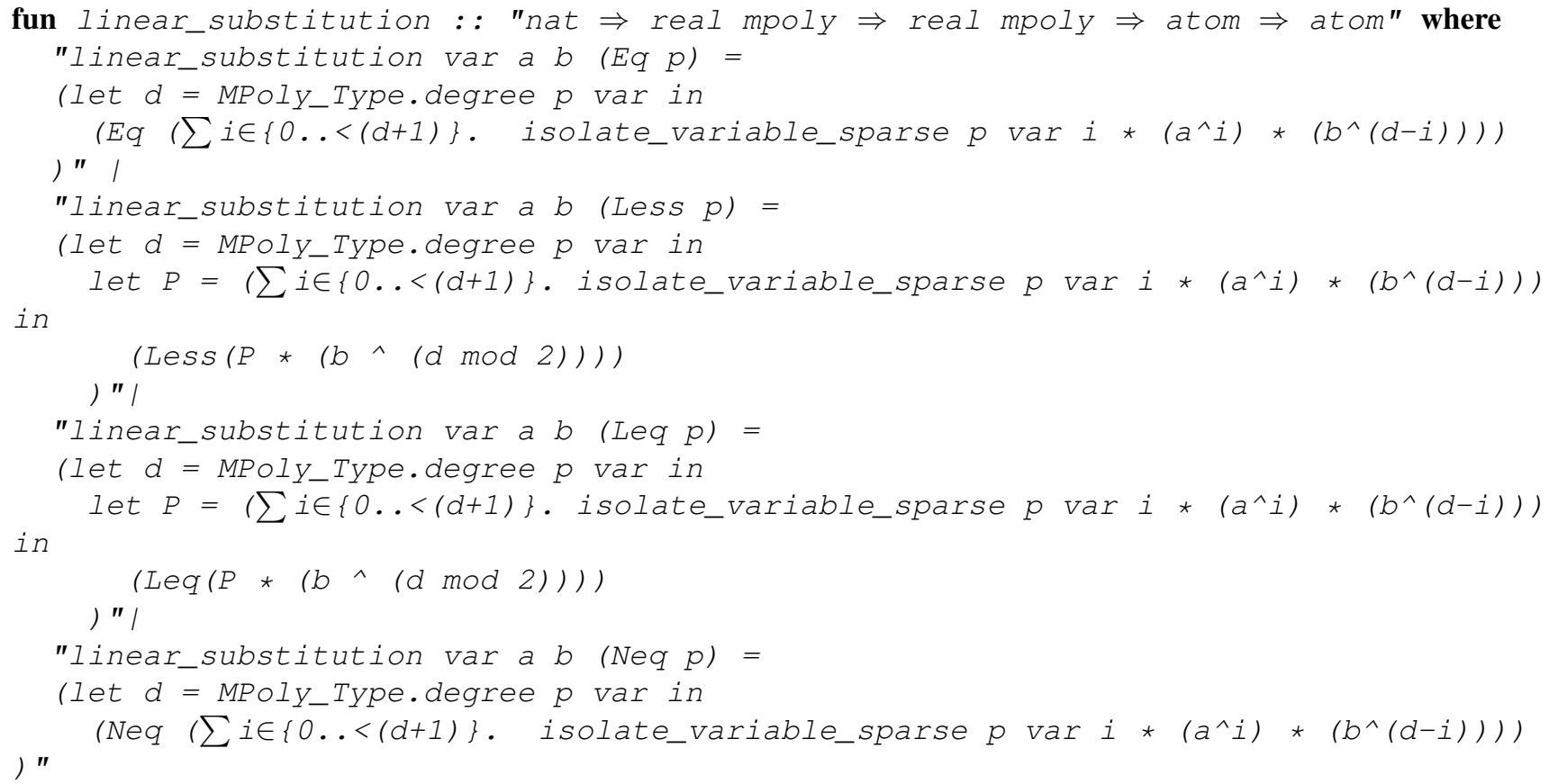

In each case, the first step of the algorithm is to recover the degree of the polynomial we wish to substitute into. We store this in $d$. Then, we can express the polynomial as a summation of monomials with respect to the variable we are eliminating on. To read off the $i$ th coefficient of the polynomial $p$ with respect to var, we use the isolate_variable_sparse $p$ var $i$ function.

For the $=$ and $\neq$ cases, we multiply the $i$ th coefficient by $a^{\wedge} i * b \wedge(d-i)$, to reflect that we have normalized by multiplying everything by $b^{\wedge} d$ (more specifically, this reflects that $\left(a^{i} / b^{i}\right) \cdot b^{d}=a^{i} \cdot b^{d-i}$ ). For the $<$ and $\leq$ cases, we additionally need to check the parity of the degree $d$. When $d$ is odd, we must multiply the whole polynomial by an additional factor of $b$, as explained in Sect. 3.2; in the code, we accomplish this by multiplying by the extra factor of $b^{\wedge}(d \bmod 2)$ in the $<$ and $\leq$ cases.

\section{A.2 Quadratic Substitution}

In Isabelle/HOL, we formalize quadratic substitution in the quadratic_sub function. Here, quadratic_sub

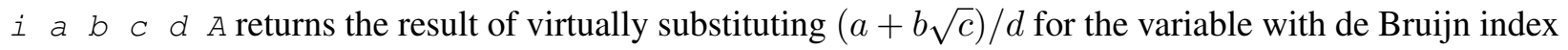


$i$ in atom $A$. All cases of the different atoms utilize the same helper functions. As explained in Sect. 3.2, they differ in the final arrangement of the polynomial $A+B \sqrt{c}$, where $A$ and $B$ are multivariate polynomials that do not mention the variable we are eliminating on.

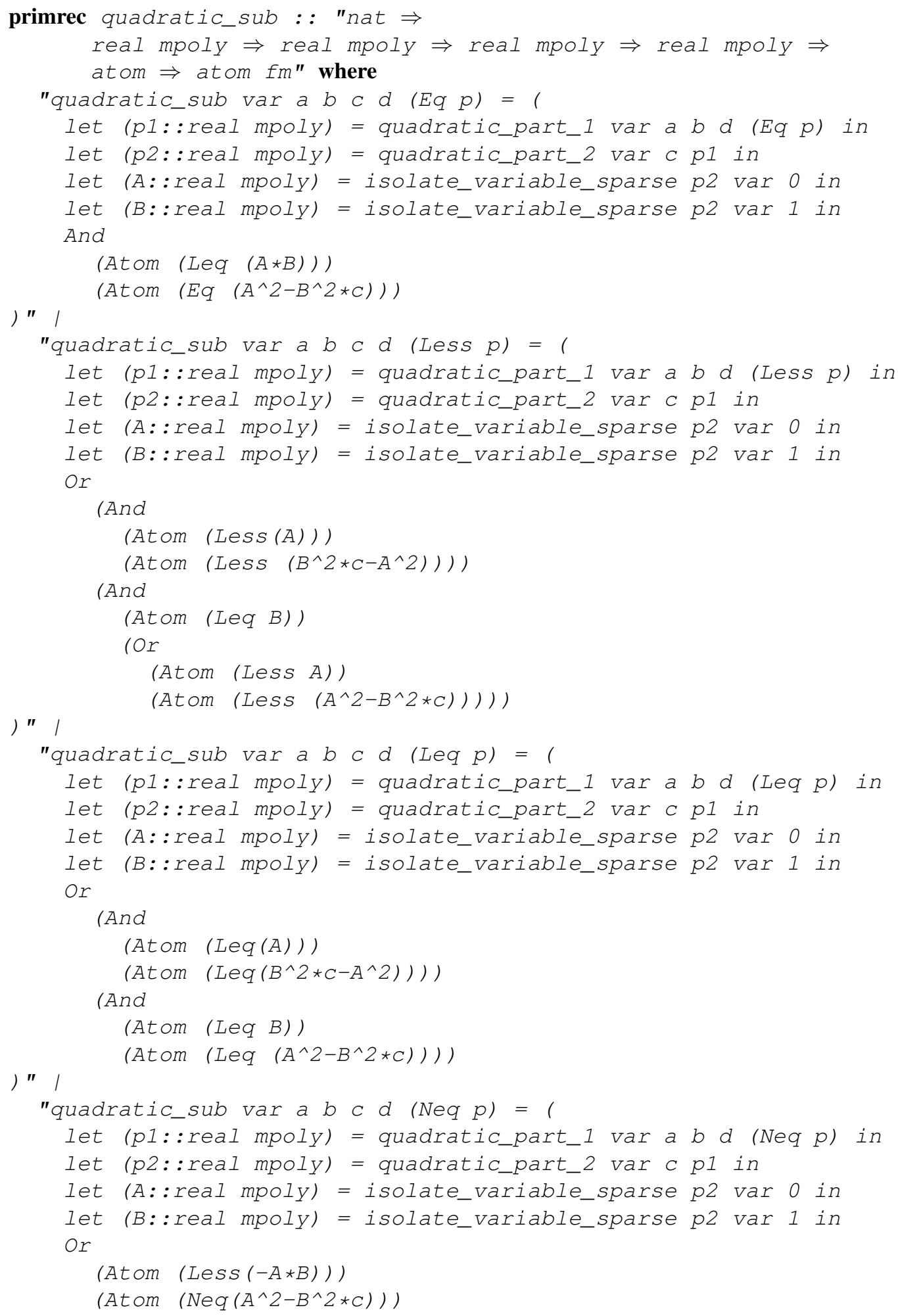


The helper function quadratic_part_ 1 formalizes the normalization of denominators discussed in Sect.3.2. It behaves identically to the Iinear_substitution function discussed in Appendix A.1, except that the fractional polynomial that quadratic_part_ 1 is substituting into variable var is $(a+b *(\operatorname{Var}$ $\operatorname{var})$ ) / $d$.

Remark 3 Notice that in the above, we are actually substituting a polynomial mentioning var for the variable var. This situation arises as a consequence of our multiple-step substitution process for quadratic roots: If we associate the polynomial variable var with the meta-variable $x$ and create a second metavariable $y$, then we want to perform the substitutions $x=(a+b y) / d$ and $y=\sqrt{c}$. After performing the first substitution for meta-variable $x$, the polynomial variable var representing the meta-variable $x$ gets eliminated. As such, it is okay for us to repurpose the same polynomial variable var to represent our new meta-variable $y$ when performing the substitution $y=\sqrt{c}$. Further, reusing this specific variable var allows us to avoid potential conflicts with other variables within the polynomial.

The helper function quadratic_part_2 handles the parity check on the normalization factor, as described in Sect. 3.2. Here, we simply collect the summation of all the even-degree monomials (in our variable of interest) into one polynomial $A$ and all the odd-degree monomials into another polynomial $B$, so that ultimately our polynomial is of the form $A+B \sqrt{c}$.

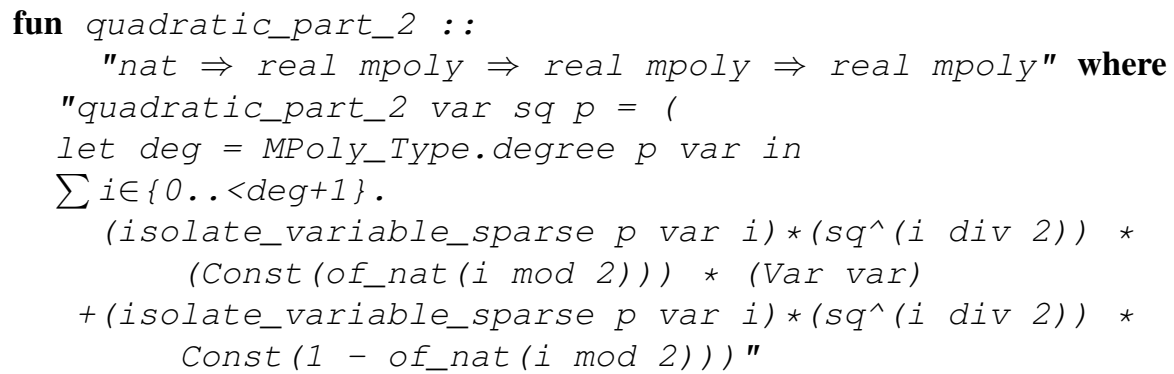

In this, of_nat casts natural numbers to real numbers. For each $0 \leq i \leq$ deg, Const (of_nat ( $i$ mod 2)) is 0 when $i$ is even and 1 when $i$ is odd. Similarly, Const ( 1 - of_nat ( $i$ mod 2)) is 1 when $i$ is even and 0 when $i$ is odd. So,

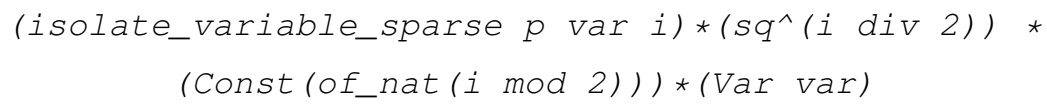

collects the summation of the odd-degree monomials (in var) in the form $a_{i} c^{\lfloor i / 2\rfloor} \sqrt{c}$ where $\operatorname{Var} \operatorname{var}$ represents $\sqrt{c}$, while

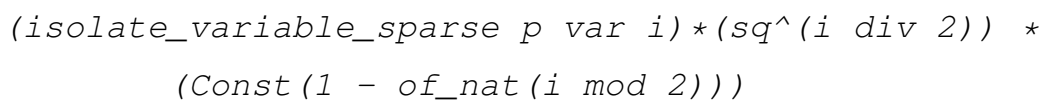

collects the summation of the even-degree monomials in var in the form $a_{i} c^{i / 2}$. Notice that in the even cases, we are able to completely eliminate the square root, whereas the odd cases leave us with a variable of degree one, for which we will need to substitute $\sqrt{c}$.

We use the technique explained in Remark 3 to handle substituting a polynomial mentioning var in for the variable var; this time, our first polynomial variable var represents the meta-variable $y=\sqrt{c}$, and after the substitution, the polynomial variable var represents the meta-variable $z=\sqrt{c}$. 


\section{B Top-Level Algorithms}

We develop (and export to SML for experimentation) several top-level algorithms that use various combinations of VS procedures and optimizations; these are briefly detailed here.

The VSLucky algorithm recursively searches through the available atom conjunct list, searching for a quadratic equality atom that features a constant coefficient with respect to the variable $x$ that we are eliminating. If it finds some $a x^{2}+b x+c$ where $a, b$, or $c$ is a nonzero constant, we are guaranteed that $a x^{2}+b x+c$ is not the zero polynomial, and thus we have the following full elimination of the quantifier on $x$, without the remaining zero case (cross reference Sect.3.2):

$$
\begin{aligned}
& \left(\exists x \cdot\left(a x^{2}+b x+c=0 \wedge F\right)\right) \longleftrightarrow \\
& \left(\left(a=0 \wedge b \neq 0 \wedge F_{x}^{-c / b}\right) \vee\right. \\
& \left.\left(a \neq 0 \wedge b^{2}-4 a c \geq 0 \wedge\left(F_{x}^{\left(-b+\sqrt{b^{2}-4 a c}\right) /(2 a)} \vee F_{x}^{\left(-b-\sqrt{b^{2}-4 a c}\right) /(2 a)}\right)\right)\right) .
\end{aligned}
$$

Then we can use VS to expand the RHS of the equivalence. So, VSLucky is indeed lucky, because it fully removes the quantifier on the variable in question.

Next, the VSEquality algorithm performs the equality version of VS (Sect.3.2) iteratively for all equality atoms of at most quadratic degree, and the VSGeneral algorithm performs the general version of VS (Sect. 3.3. 8

Finally, we have VSLEG which performs all three of VSLuCky, VSEquality, VSGeneral (in that order, as the first is the quickest while the last is the most general); this algorithm was the most competitive one on our benchmarks.

We now discuss the correctness theorem for these algorithms.

\section{B.1 Correctness of Top-Level Algorithms}

The correctness of our top-level algorithms is established by the following theorem, which expresses that any initial formula $\varphi$ is logically equivalent to the formula obtained by running the algorithm on $\varphi$; in other words, the two formulas (initial and final) have the same truth-value in every state, i.e. for every valuation of free variables.

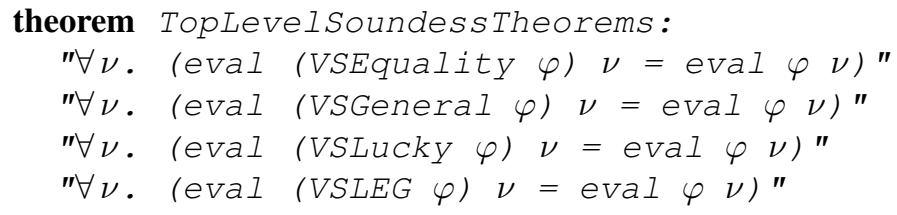

In this theorem, our eval function captures the semantics of substituting a valuation into a formula: For a valuation characterized by a list $\nu$ of real numbers and a formula $\varphi$, eval $\varphi \nu$ is true whenever $\varphi$ is true at $\nu$, i.e. when the $i$ th entry of $\nu$ is plugged in for the $i$ th free variable of $\varphi$ (for all $i$ ). More precisely, the exact definition of eval is as follows:

\footnotetext{
${ }^{8}$ Additionally, we have VSEquality_3_times, which performs the equality algorithm three times, and VSGeneral_3_times, which performs the general algorithm (respectively) three times. Although these account for the kind of potential edge cases discussed in Sect.4.2.2 they did not realize significant experimental benefits over VSEquality and VSGeneral.
} 


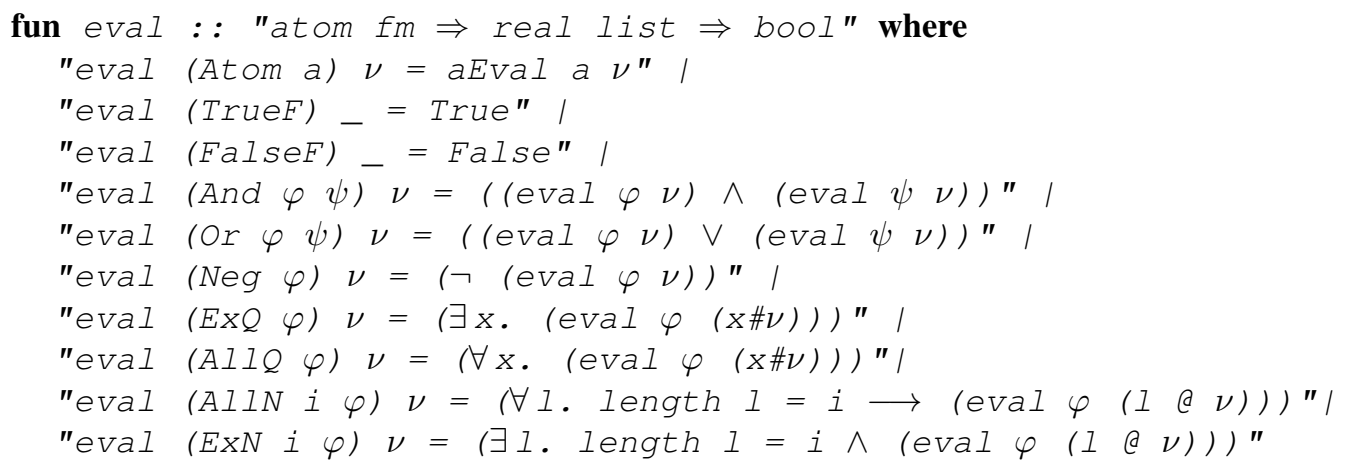

This is the canonical semantics for evaluating atom formulas. The interesting cases are the Atom, ExQ, and $A I l Q$ cases. We discuss each.

In the Atom case, the aEval function is as follows:

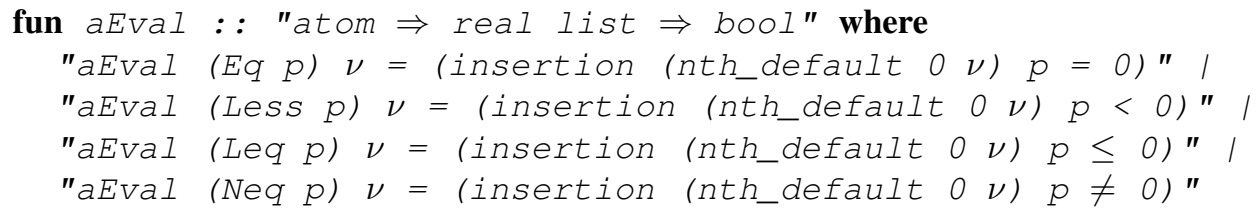

Here, we are using the insertion function from the multivariate polynomials library [26] to insert the $n$ values from list $\nu$ for variables $0, \ldots, n-1$. This captures evaluation by substituting values for the free variables in the formula.

Because the first argument of the insertion function has type (nat $\Rightarrow{ }^{\prime} a$ ), where in our case ' $a$ is real, we use Isabelle/HOL's standard library function $n t h$ default to expand $\nu$ into a mapping from nats to reals. That is, (nth_default $0 \nu$ ) $i$ is the $i$ th element of $\nu$ when $i$ is less than the length of $\nu$ (lists are 0 -indexed in Isabelle/HOL), and 0 otherwise. Note that this means that if our list $\nu$ is not long enough to cover the valuations for all the free variables in the polynomial, the $n t h$ _default function will assign it a value of 0 . This is merely a convenience; our correctness lemmas are unaffected by this, as they quantify over all possible valuations $\nu$ (and thus over all valuations of the correct length).

The $E x Q \varphi$ case of a new existential quantifier is handled by embedding into Isabelle's built-in notion of existential quantification over the real numbers. More precisely, in $\exists x$. (eval $\varphi(x \# \nu)$ ), Isabelle treats the quantified variable $x$ as a new free variable, which is added to the front of the valuation $\nu$, written $x \# \nu$. The $A I I Q$ case is similar.

As an example, consider evaluating

$$
\exists x . \forall y \cdot(x+y \cdot y+z>0)
$$

in a state $\nu$ where $\nu(z)=1$. In our framework in Isabelle/HOL, this translates as:

$\operatorname{eval}(\operatorname{ExQ}(\mathrm{All} Q(\operatorname{Var} 1+\operatorname{Var} 0 * \operatorname{Var} 0+\operatorname{Var} 2>0)) 1$

Here we have two quantified variables, Var 0 and Var 1, and one free variable, Var 2 (this is because we are using de Bruijn indices). Var 0 matches the $A \perp \perp Q$ quantifier and Var 1 matches the ExQ quantifier. We are considering the valuation where Var 2 is set to 1.

In the first step, we expand to:

Exists $\mathrm{x}$.

(eval (Alle (Var $1+\operatorname{Var} 0 * \operatorname{Var} 0+\operatorname{Var} 2>0))(x \# 1)$ ), 
and in the second step, we achieve:

Exists x. Forall y.

(eval (Var $1+\operatorname{Var} 0 * \operatorname{Var} 0+\operatorname{Var} 2>0)(y \#(x \# 1)))$.

This asks whether there exists an $x$ such that for all $y, x+y^{2}+1>0$, which matches the desired semantics.

We additionally include $E X N$ and $A I I N$, which take in two inputs $i$ and $\varphi$ and are equivalent to the single quantifier forms $E \times Q$ and $A I I Q$ repeated $i$ times on $\varphi$ (which, thanks to de Bruijn indices, are quantifiers for $i$ different variables). In the eval function, these are represented via a quantified list $I$ of real number valuations of length $i$. Having these $E X N$ and $A I I N$ in the representation allows for specialized algorithms like our block quantifer heuristics (see C.3).

Now that we have established that our correctness theorem is correctly stated, we discuss its proof.

\section{B.2 Proving Correctness: Extensibility}

It would be very tedious to independently prove the correctness theorems for all of our top-level algorithms. Instead, our framework is designed so that any optimization opt can easily be incorporated into our framework, as long as opt does not change the truth-value of any formula; i.e. our framework is designed to be extensible. This allows us to cleanly substitute different combinations of optimizations into our top-level QE algorithms without incurring the burden of significant reproving.

This extensibility can be seen in the following correctness lemma for our $Q E \_d n f$ function, which lifts QE algorithms defined for a single quantifier to apply to all quantifiers using the modified DNF transformation discussed in Sect.4.2.

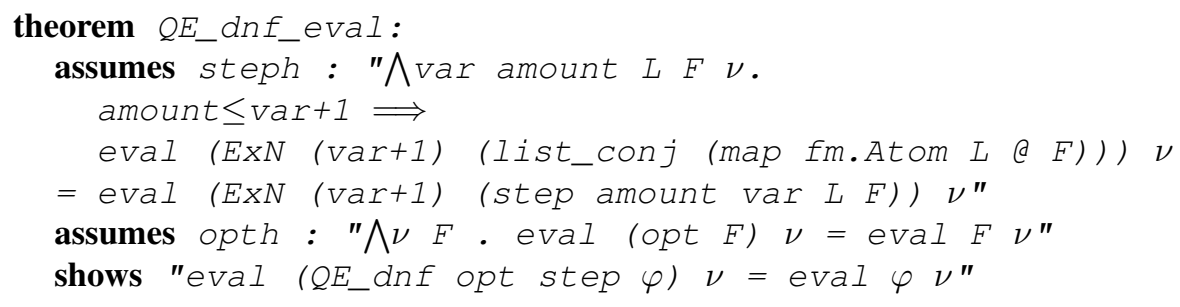

Here, the step function is intended to be a function that performs virtual substitution. Its behavior is governed by the steph hypothesis. The opt function is intended to be a function that performs various optimizations, and its behavior is governed by the opt hypothesis. Intuitively, $Q E \_d n f_{-}$eval proves that any function that obeys the steph hypothesis (as we prove that our VS procedures do) and any functions that obey the opt hypothesis (as we prove that our optimizations do) can be combined to create an overall top-level QE procedure. We now discuss the characteristics of opt and step further.

The opth hypothesis assumes that we have a procedure opt which preserves the truth value of the evaI function for every valuation on every formula. In our QE framework, this function is called before performing the DNF transformation of Sect.4.2.3, and then step is called after performing the DNF transformation.

As specified by the steph hypothesis, the step function receives as input two natural numbers amount and var, a conjunct list of atoms, $L$ and a conjunct list of atom formulas, F. Here, amount is optional information that indicates for how many of the var+1 existential quantifiers in the prefix QE has not yet been attempted. Since the modified DNF transformation is performed before step, some quantifiers will result from this algorithm, and for these we have already attempted VS. Tracking amount allows us to stop computation early, rather than re-attempting futile VS on quantifiers where it previously did not apply. 
The left-hand side of the equality in steph represents the form that our formula takes in one of the disjuncts of the DNF transformation (cross reference Sect.4.2.3): there are var+1 existential quantifiers in this disjunct (we use zero-indexing for the variable), and everything in $L$ and $F$ is conjuncted. Intuitively, this is what we pass into virtual substitution. The right-hand side of the equality in steph represents the result of calling the step function. The equality captures that the original formula and the formula we obtain by applying step have the same truth value in any valuation $\nu$. So, overall, the steph equality captures that step can perform any arbitrary manipulation of the inputs as long as it preserves logical equivalence.

The $Q E \_d n f \_e v a I$ lemma showcases the extensible nature of our framework and allows us to determine the soundness of the top level algorithms described in Appendix B, but it makes no claim about whether VS is actually simplifying our formula. We are unable to make such claims for this top-level procedure, as we must allow for our VS algorithms to fail to make progress in cases where VS does not apply (e.g. in the presence of high degree polynomials), but we now discuss the specific cases where our VS step procedures successfully eliminate quantifiers, as well as the important correctness lemmas for these procedures.

\section{B.3 Proving Correctness: VS}

Our elimVar function is the multivariate analog of the univariate general VS procedure discussed in Sect.3.3.3 (and it closely resembles this procedure). More specifically, elimVar is an overarching proof procedure that analyzes the roots of a polynomial and applies the appropriate VS algorithms for both the equality and the off-root cases. It checks whether we wish to substitute the exact root (for $=$ and $\leq$ atoms) or the off-roots (for $<$ and $\neq$ atoms).

As input, elimvar receives a variable var we are eliminating on, a list of atoms $L$ and formulas $F$ which are joined by conjunction, and the atom we wish to substitute, which is guaranteed to be at most quadratic (cross-reference Sect.4.2.3). Each of the top-level VS algorithms utilizes el imVar as a helper function to substitute particular atoms. Significantly, elimVar has the property that it removes the variable it is substituting. This is expressed in the following lemma, where our variableIsRemoved function is true when the input variable var is not present in a formula:

lemma elimVar_removes_variable: "variableIsRemoved var (elimVar var L F At)"

An important lemma for the equality case of VS can be proven when we specify that the atom At input to the elimVar function is an equality atom with a quadratic or linear polynomial with respect to the variable being eliminated:

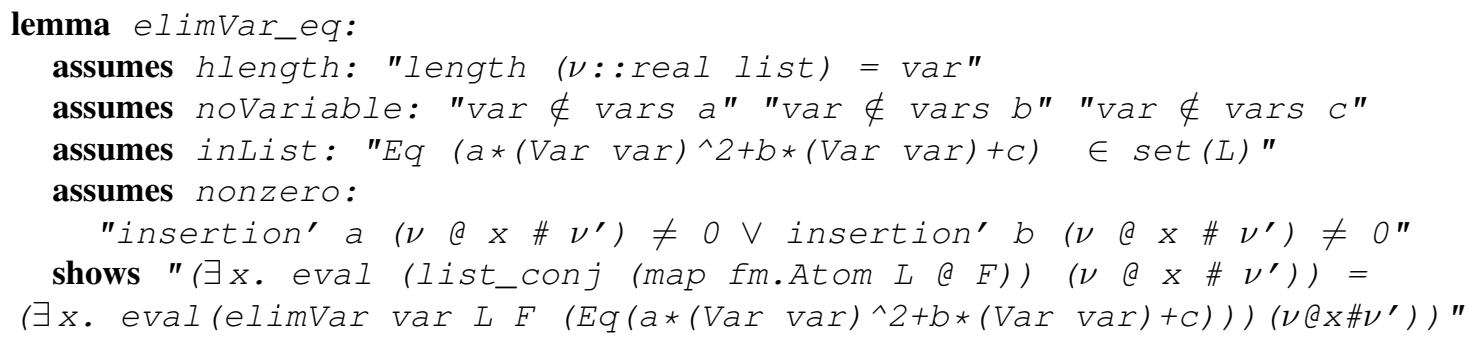

Here, we are assuming that we have the equality atom $a *(\operatorname{Var} \operatorname{var})^{\wedge} 2+b *(\operatorname{Var} \operatorname{var})+c$ within our list of atoms $L$, where the variable var does not occur in the coefficients $a, b$, and $c$; this is captured by the inlist and novariable hypotheses. We also assume that this polynomial is quadratic or linear in the particular variable of interest (in the nonzero hypothesis). Performing elimvar on this atom allows us to remove the quantifier completely: in the linear case, when $E q\left(a *(\operatorname{Var} \operatorname{Var})^{\wedge} 2+b *(\operatorname{Var} \operatorname{Var})\right.$ 
$+c)$ is $E q(b *(\operatorname{Var} \operatorname{Var})+c)$, elimVar virtually substitutes $-c / b$ for var into every formula; in the quadratic case, elimVar produces the disjunct of virtually substituting the two possible quadratic roots into every formula. This lemma establishes the correctness of the Equality VS algorithm as described in Section 3.2. It notably only establishes equivalence of existence of a value for the variable $x$, rather than equivalence of truth-value.

Additionally, we use el imVar to formulate the lemma for the general VS case:

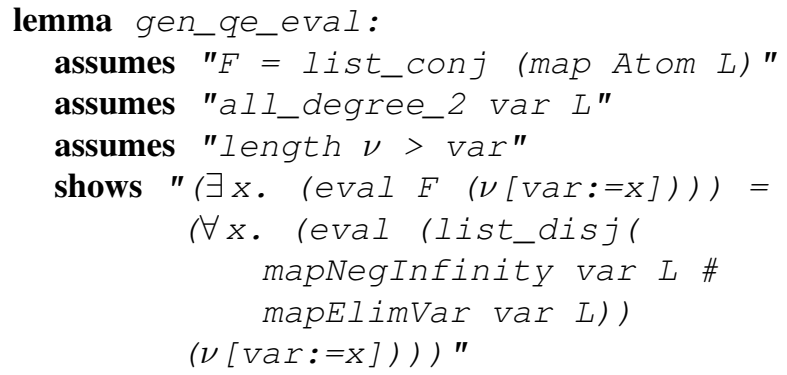

This lemma regards the executable multivariate procedure that corresponds to the univariate lemma explained in Section 3.3.3.

Here, we assume that our formula $F$ is a conjunction of atoms $L$ as expressed in the first hypothesis. Additionally, the second hypothesis expresses that $F$ has the requisite shape for general VS to apply; this means that every polynomial in $L$ has at most degree two with respect to the variable we are eliminating, var. The third assumption states that our valuation $\nu$ is long enough to cover the variable in question (which makes it a valid valuation).

Under these assumptions, we show that there exists an $x$ that can be substituted for var to make formula $F$ true iff substituting one of the sample points prescribed by virtual substitution (which are negative infinity, the roots of the atoms with respect to var, and the corresponding off-roots) for var makes $F$ hold. Notice that the $\forall$ quantification on the $x$ on the RHS of the equality is permissible because we have actually eliminated the variable $x$ from the formula after applying virtual substitution (this is established by the elimvar_removes_variable lemma).

\section{B.4 More Polynomial Library Contributions}

In order to prove correctness of VS, we needed to formalize a large number of additions to the Isabelle/HOL multivariate polynomials library. For example, we added a partial derivative function (needed to formalize VS for infinitesimals, as discussed in Sect.3.3.2); and prove its correctness for polynomials of degree at most two (because more is not needed for quadratic virtual substitution).

For execution, we also needed to extend code theorems for the generically defined insertion function, which inserts a valuation into a polynomial, in order to be able to compute the result of valuations and export our program. This required a number of lemmas that rely on the monomials function, which separates a polynomial into a sum of monomials, and the degree function, which computes the degree of a multivariate polynomial with respect to a single variable.

To make these new functions usable in proofs, we formulated a large collection of lemmas for polynomials with real-valued coefficients. These include a variety of simplification lemmas for the interaction of the isolate_variable_sparse function discussed in Sect.4.4 and the insertion, and degree functions across summations and products of polynomials.

To utilize the variables within polynomials as de Bruijn indices, we implemented various lifting and substitution operations. Our liftPoly function takes in a lower limit $d$, a lifting amount $a$, and a polynomial 
$p$ and returns a polynomial which reindexes variables within $p$ such that every variable greater or equal to $d$ is increased by $a$. This is commonly denoted by $p \uparrow_{d}^{a}$. This liftPoly is needed in cases where we want to reshuffle formulas to increase the number of quantifiers surrounding a formula. For example, $\forall .((\exists A) \wedge B)$ is equivalent to $\forall \cdot \exists .\left(A \wedge\left(B \uparrow_{1}^{1}\right)\right)$.

We also need an inverse function to liftPoly, which we call lowerPoly. Whenever we have eliminated a quantified variable with our QE procedure, we can drop that quantifier and use lowerPoly to reindex all the other variables accordingly. Our clearQuantifiers procedure implements this.

\section{Optimizations}

We formalize a number of optimizations for VS, as optimizations are critical for achieving reasonable performance. It will take some time to catch up to the highly optimized performance of tools like Wolfram Engine and Redlog.

This is the benefit of an extensible framework: future optimizations can be easily integrated. From Appendix B.2, we see that any optimization function opt that satisfies the truth-preservation lemma lemma "eval (opt F) $x S$ = eval F xS" can be cleanly integrated into our algorithm (using the QE_dnf_evaI theorem), and the composition of several of these optimization functions directly preserves this property. We discuss our current optimizations in this appendix.

\section{C.1 Unpower}

As our algorithm performs VS on quadratic and linear polynomials, it is critical to reduce the degree of polynomials whenever possible. The most natural simplification we can perform is to factor out a common $x^{n}$ from every monomial:

$$
\sum a_{i} x^{n+i}=x^{n} \sum a_{i} x^{i}=p x^{n}
$$

From here, we can split the atom $p x^{n} \sim 0$, where $\sim \in\{=,<. \leq, \neq\}$, into lower-degree atoms that involve $p$ and $x^{n}$ separately.

In the equality case, $p x^{n}=0$ reduces to $x=0 \vee p=0$, as the product of the components is zero if and only if at least one of the components is zero. For inequalities, we case on the parity of $n$. If we have an even exponent, we can reduce $p x^{n}<0$ into $p<0 \wedge x \neq 0$, since $x^{n}$ is nonnegative. Otherwise, we must assert that the sign values differ: $(p<0 \wedge x>0) \vee(p>0 \wedge x<0)$.

All $\neq$ are treated as negated equality atoms. For $\leq$ atoms, we follow a similar structure to $<$ : when $n$ is even, we result in $p \leq 0 \vee x=0$, and when $n$ is odd we result in $p=0 \vee(p<0 \wedge x \geq 0) \vee(p>0 \wedge x \leq 0)$.

\section{C.2 Simplifying Constants}

It is clear that the atom $5=0$ is always false and can be replaced by the FalseT formula. Our simpfm function replaces constant polynomial atoms with their respective TrueT or FalseT evaluations and performs shortcut optimizations for the $\vee, \wedge, \neg$ connectives. This is especially useful when our QE algorithm is successful on closed formulas: since no more variables are present, the formula becomes a collection of constant polynomials atoms and connectives which means the whole formula can be reduced to either TrueF or FalseF with this simplification. 
This constant identification and constant folding is also crucial for effiency within the QE procedure to cut down the expansion of the formula. Recall that we transform QE problems to have the form

$$
\bigvee \exists z_{0} \cdot \cdots \exists z_{n} \cdot \exists x \cdot\left(\left(\bigwedge A_{i}\right) \wedge\left(\bigwedge \forall y \cdot F_{j}\right)\right)
$$

where the $A_{i}$ are atoms and the $F_{i}$ are formulas (see Sect.4.2.3). Now consider a QE problem of the form $\exists x .\left(a x^{2}+b x+c=0 \wedge F\right)$. In the event that at least one of $a, b$, or $c$ is a nonzero constant polynomial, we can immediately determine the only possible values of $x$ in the whole formula, which are just the roots of this specific polynomial. As such, in both our general QE and equality QE algorithms, for each disjunct in a formula of the form of (6), our algorithm performs a linear scan for these "lucky" atoms within the conjunct list of atoms before proceeding with the VS algorithm. If a lucky atom is found, we immediately eliminate its associated quantifier and then proceed with other steps of the algorithm. This optimization demonstrates significant experimental benefits, as it eliminates quantifiers without greatly increasing the size of the formula. It also utilizes the DNF form optimizations discussed in Section 4.2.3 to reach underneath existential quantifiers to find more "lucky" atoms. It could be further adapted in the future to, for example, allow for simplifications underneath universal quantifiers.

Even better than the "lucky" atoms, where a single coefficient is constant, are "luckiest" atoms, in which all coefficients are constants: with luckiest atoms, we are substituting real numbers rather than polynomials. We found that "luckiest" atoms are empirically very significant (identifying and cleverly utilizing them yields significant speedup), so our algorithms preprocess input formulas for these kinds of atoms and perform virtual substitution on them first.

\section{C.3 Variable Ordering Heuristics}

Variable ordering heuristics are of great practical significance in QE [16]. We proved that one can freely swap the ordering of quantifiers in a homogeneous block (a number of existential or of universal quantifiers that occur in a row) without affecting a formula's truth value. To capitalize on this, our groupQuantifiers function locates instances of homogeneous blocks in a formula and converts them into an equivalent block

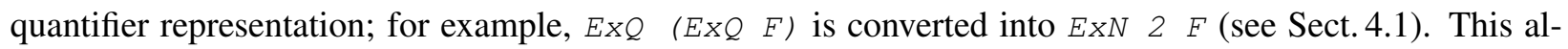
lows later algorithms to identify blocks by pattern matching. When the VS algorithm reaches a block ExN $n F$, it uses the modified DNF algorithm on $F$ and focuses on a single disjunct, at which point we invoke a heuristic function to choose which of the quantifiers in the innermost block to eliminate first.

Since DNF yields a disjunct list of conjuncts, the input information to the heuristic function (at each disjunct) is a conjunct list of atoms $L$ and formulas $F$. As such, a heuristic function $H$ is of the type $H:$ : nat $\Rightarrow$ atom list $\Rightarrow$ atom $\mathrm{fm}$ list $\Rightarrow$ nat, where $H \quad(N-1) \quad L \quad F$ analyses the input conjuncts $L$ and $F$ and determines the optimal variable ranging from $O$ to $N-1$ to eliminate on (remember that we use 0 -indexing for variables). After selecting a variable var to eliminate, we swap var with variable $N-1$, perform VS on the newly reindexed var, optimize the result, and then run DNF again. We then recursively proceed on each new disjunct until we have attempted to eliminate every quantifier.

The only property that the heuristic function must satisfy for correctness purposes is that it must suggest a variable within the block of interest; this allows users to create their own heuristics without incurring significant proof burden. Verifying this property is trivial for heuristics that check their result to disallow results outside of the desired range.

We implement three heuristic functions: one of our own design, one based on the literature, and the identity heuristic. The identity heuristic always returns the innermost variable, which yields experimental results comparable to an earlier version of the framework which did not implement block quantifiers; this 
demonstrates that we do not incur significant overhead by supporting block quantifiers. We also implement a heuristic based on Brown's heuristic for quantifier ordering for CAD, as presented in [9]; this yielded promising experimental results. Lastly, the heuristic that we designed both chooses which variable to eliminate first and also chooses which VS algorithm to use at each step; this heuristic was the most experimentally successful of the three. Overall, our results indicate that block quantifiers do not introduce significant overhead and confirm that variable ordering heuristics are of practical significance in virtual substitution. 